\section{anthropology} \& materialism

\section{Anthropology \& Materialism}

A Journal of Social Research

$2 \mid 2014$

The Persistence of Myth

\title{
Le chant des entrailles, une philosophie de l'intestin
}

\section{Mahoro Murasawa and Stéphane Nadaud}

\section{(2) OpenEdition}

\section{Journals}

\section{Electronic version}

URL: http://journals.openedition.org/am/353

DOI: $10.4000 / a m .353$

ISSN: 2364-0480

\section{Publisher:}

CETCOPRA, CRASSH - Center for Research in the Arts Social Sciences and Humanities, Fakultät

Gestaltung - Universität der Künste Berlin

Electronic reference

Mahoro Murasawa and Stéphane Nadaud, « Le chant des entrailles, une philosophie de l'intestin », Anthropology \& Materialism [Online], 2 | 2014, Online since 15 April 2014, connection on 01 May 2019.

URL : http://journals.openedition.org/am/353 ; DOI : 10.4000/am.353

This text was automatically generated on 1 May 2019.

Tous droits réservés 


\title{
Le chant des entrailles, une philosophie de l'intestin
}

\author{
Mahoro Murasawa and Stéphane Nadaud
}

«Dans le plus profond de la mer la plus profonde,

j'ai creusé un trou dans le sein même de mon propre corps sphérique. Parce que je voulais manger plus. Parce que j'avais faim. À partir de ce trou central, j'ai percé mon corps en deux extrémités afin qu'il devienne un tube. Parce que

je voulais manger plus, parce que ma faim ne s'apaisait jamais. J'ai alors pris (in)conscience de devenir « intestin ». Pour manger plus encore j'ai même réussi à fabriquer toute une organisation complexe qui me permit alors de me mouvoir. Je suis devenu un tube avec un système nerveux moteur. Et plus je voulais manger, plus ma faim gagnait en puissance, plus le système nerveux s'agrandissait. Un jour, le contrôleur est apparu dans le système nerveux : il s'est auto-appelé « cerveau ». C'est alors que j'ai acquis la possibilité de prédire l'avenir et que je me suis retrouvé attaché à un roc au plus haut de la plus haute montagne, une partie de mon tube éternellement dévoré par un aigle. » Journal de Prométhée, 12000 A.C.

\section{(0). Prolégomènes à une philosophie de l'intestin}

1 Dans l'introduction que vous avez dans les mains, sous les yeux, tout est contenu - comme un intestin à l'intérieur d'un intestin. Il se veut l'introduction d'un livre à venir - merci M. 
Blanchot -, immodestement titré Tout sur Tout. Les affirmations y sont donc affirmatives, péremptoires certaines fois, laconiques souvent mais jamais modestes - à l'image de l'énigmatique exergue de la précédente phrase à laquelle nous donnerons bientôt quelque sens. C'est qu'elle ouvre, cette somme d'affirmations, à tout l'intérieur (et l'extérieur) des entrailles, des intestins que sont ce livre : aussi, celui qui s'engage dans les villosités de cet intestin, s'il trouve cette introduction à certains moments peu étayée ou justifiée (pas d'appel de notes dans cette introduction : tout est dans le texte, dans ou entre ses mots), aura-t-il le loisir de plonger plus avant encore dans ledit intestin - le livre à venir - que subdiviserons en bolges (de l'italien bolgia, utilisé par Dante dans son Enfer: les trous ou les sacs où sont entassés, au huitième cercle, les damnées - les physiologistes disent villosités). Ces bolges permettront, au gré des envies et des nécessités, de comprendre la construction de cette introduction, d'en développer ou d'en approfondir les soubassements ou les extensions (de la cave au grenier), et ont surtout comme vocation de poursuivre celles-ci comme autant de lignes de fuite.

2 Cette introduction, comme son titre l'indique, parle des entrailles et de l'intestin. Autrement dit, elle parle de physique, au sens le plus matériel du terme: la physique entendue comme synonyme du corporel. Par corporel, nous entendons les entités que les philosophes Deleuze-Guattari, s'inspirant d'un autre philosophe (français comme eux) nommé Simondon, ont dit de ne pas séparer en vivant (plus ou moins doté d'une âme, d'une subjectivité) d'un côté, et en objet inanimé (sans âme) de l'autre. C'est notre définition de la physique qui, nous le verrons dans cette introduction, est loin d'être celle du commun des philosophes (fussent-ils français ou japonais). Comme Guattari-Deleuze, nous pourrions les appeler, ces corps, machines. Car, une fois déposée la distinction entre le vivant et le non-vivant, nous ont-ils appris, la physique peut être pensée en termes de machines. En fait, ce que nous proposons avec cette introduction qui parle d'intestin, c'est d'en finir avec l'opposition - le complot? - systématiquement opéré entre physique et métaphysique.

3 Les éditions classiques des livres du philosophe grec Aristote, faites par Andronicos de Rhodes, placent «tout naturellement» la métaphysique après la physique - le cerveau (que l'on dit en japonais nô) après l'intestin comme tube digestif (chô). C'est que le cerveau (qui, dans sa conception occidentale, se veut si peu physique - malgré ses synapses, ses neurones, etc. - et si idéal puisque c'est lui, le cerveau, qui est sensé justement s'occuper de la métaphysique) arrive après l'intestin, comme tube digestif, (que la même philosophie occidentale considère au contraire comme une chose toute physique, toute machinique, voire même sale et triviale). Mais venir après ne signifie nullement remplacer. Contrairement à ce que peut affirmer le biologiste Michael Gershon, le cerveau et l'intestin comme tube digestive sont deux entités hétérogènes, irréductibles l'une à l'autre. Lorsqu'il affirme, dans son best seller que le tube intestinal est un «deuxième cerveau ", il ne fait que poursuivre cette bévue philosophique qui consiste à faire de la physique une composante (une condition) de la métaphysique, celle-ci étant bien entendue supérieure à celle-là, cette dernière coiffant cette première : l'intestin, comme machine physique, comme tube digestif, devient le lieu de la merde, du corps bassement vulgaire (avec ses orifices, ses excrétions, ses pratiques sodomitiques perverses...) et le cerveau arrive à en oublier qu'il est lui-même un organe, une machine, et se transforme, se sublime en un idéal éthéré où rien de vulgaire et de bas ne saurait s'exprimer.

4 En écrivant cette introduction, nous avons pensé dans un premier temps que le cerveau était un prolongement de l'intestin, que le cerveau était de l'intestin qui se complexifiait, 
qui se prolongeait à la manière d'un repliement infini sur lui-même - un pli de l'intérieur, comme on retourne un gant - même si ce pli le fait s'étendre à l'infini pour envahir le cosmos tout entier. Mais nos recherches embryologiques, et nos enquêtes esthétiques et politiques - bref : notre façon de faire de la philosophie - nous ont convaincus que nous faisions fausse route. En effet : si c'est une erreur de croire que le cerveau serait une idée transcendantal qui dépasserait l'intestin comme tube digestif - vision tristement métaphysique (Aristote) ; si c'est une erreur de croire que le tube intestinal, parce qu'il est aussi composé de synapses et autres neurotransmetteurs, serait comme le cerveau mais « second» tout de même - (Gershon); si nous critiquons d'emblée ces penseurs qui subordonnent la physique à la métaphysique, il nous a fallu nous faire violence pour ne pas faire la même chose, mais à l'inverse, et soumettre la métaphysique à la physique (notamment en affirmant que le cerveau serait encore de l'intestin).

\section{Antidualisme}

Prenons par exemple le physiologiste japonais Fujita Tsuneo. Il pense que l'ancêtre du cerveau, c'est l'intestin. Son livre, non traduit en français, mais dont la moelle se trouve, si le lecteur intéressé souhaite la chercher, dans les villosités de cette introduction, son essai donc, Chô ha kangaeru, nous dit que "L'intestin pense ». En étudiant les hydres, qui sont des organismes très archaïques toujours présent sur terre mais qui n'ont pas évolué depuis 500 millions d'années, il montre que les cellules qui formeront chez les organismes plus récents les neurones, sont des dérivés des cellules de l'appareil intestinal (qu'il nomme les paraneurones). Par cette étude physiologique il souhaite prouver que, chez l'homme, la pensée du cerveau est matérielle au même titre que la digestion l'est pour le tube digestif. Cette pensée matérialiste, qui nous a séduit dans un premier temps, nous laisse néanmoins perplexe. En effet, il semblerait que Fujita ne parvient à sortir de la dualité que nous réinterrogeons. D'une certaine manière Fujita est une sorte d'antiGershon et au livre de ce dernier idéaliste (Second Brain, your Gut has a Mind of its own), le livre de ce premier matérialiste aurait pu se nommer: Second Gut: your Brain has a Digestion of its own. Autre exemple: Marx? Pourrait-on, de la même façon, en faire un anti-Aristote? Un philosophe qui aurait, pour sa part, mis le chapitre sur la physique après le chapitre sur la métaphysique. Bref, là où nous ne pouvons pas suivre Aristote dans son idéalisme, nous avons le même mal à suivre Marx dans son matérialisme. Nous le ferons, mais, pour le moment, nous n'en avons pas ni la force ni le courage. Peut-être parce qu'une telle question (qu'est-ce que le matérialisme de Marx, et en quoi nous insatisfait-il) se laisse difficilement résumer ou expliquer dans une introduction.

Bref, tout cela reste très dualiste... Nous, nous sommes au mieux pragmatiques, au pire vitalistes. Tout cela est en fait très simple : si nous pensons que tout est intestin, car pour nous tout est physique, tout est machines, et que, par là même, nous pensons le cerveau et le tube digestif comme deux machines (voire peut-être même une seule machine), nous ne commettrons pas l'erreur, que nous ont pourtant enseignée tous nos maîtres en philosophie (sauf, peut-être, Nietzsche ou Kûkai), de mettre le cerveau sous l'égide du tube digestif, de mettre la métaphysique à genoux devant la physique. La métaphysique, nous la laisserons là où elle est : nulle part. 


\section{Un intestin/plan de consistance}

7 Nous ne poursuivrons donc pas la tradition philosophique en construisant une nième physique qui serait la condition de la métaphysique [un intestin tube digestif (chô) soumis à (et différencié d'un) cerveau (nô)] ; et nous ne nous croirons pas subversifs en prônant une métaphysique humiliée devant la physique [où tube digestif (chô) et cerveau (nô) sont tout autant différenciés]. Mais nous œuvrerons pour une physique sans métaphysique, se saisissant tout autant du cerveau que de l'intestin, d'une machine que nous pourrions appeler en français, cervintestin, mais que, devant la laideur de ce néologisme, nous avons eu la tentation de qualifier d'un mot-valise japonais: le chônô (agglutination des caractères d'origine chinoise signifiant intestin et cerveau). Mais qu'avons-nous besoin de néologismes? Les intestins ne sont-ils pas, au bout du compte, les meilleurs exemples de ce qu'est, pour nous tous, un corps physique - et c'est bien de Physique dont nous voulons parler - une physique sans métaphysique ou, plutôt, une physique par-delà la distinction entre physique et métaphysique. Mais prenons garde : il ne l'est pas, physique, parce qu'il contient de la merde ou parce que il est un tube, il l'est parce que, comme le disent Gershon et Fujita, il contient et des neurones et de la merde tout comme le cerveau, lui aussi, contient et ses neurones et sa merde - ce qui fait du cerveau et de l'intestin des organes.

8 Alors pourquoi, nous interrogera-t-on peut-être, ne pas titrer notre ouvrage Le chant des entrailles, une philosophie du cerveau? Parce que nous croyons que l'intestin est avant tout le nom d'un mouvement plus que d'une forme. L'organe que nous connaissons sous le nom d'intestin, le tube digestif, a dérobé son nom à ce mouvement: c'est même la philosophie occidentale, en construisant le dualisme, qui est le véritable coupable de ce vol. Si l'intestin est d'abord le plan où a consisté, pendant toute l'histoire de la philosophie occidentale, ce que nous avons analysé comme dualisme (métaphysique / physique ; cerveau / intestin comme tube digestif), il est en réalité infiniment plus grand (et infiniment plus petit) que cela : le dualisme n'est que le rabattement de ce plan qui, si on le laisse s'exprimer, peut ouvrir à tous les possibles possibles : il est ce que DeleuzeGuattari appellent un plan de consistance, un champ pré-philosophique, pré-artistique, pré-scientifique. Il est le ciel et la terre ensemble, où ciel et terre ne se distinguent pas. Il est un et qui est aussi un ou, un ou non exclusif, un espace sans espace où le monde est la terre et le ciel sans être l'un ou l'autre, mais tout en étant différenciés quand même, un temps sans temps où l'humanité est homme et femme, parent et enfant, sans être les uns ou les autres, mais tout en étant différenciés quand même. C'est la raison pour laquelle nous ne pouvons pas vraiment imaginer ce qu'est l'intestin, car il est devenir puisque mouvement. Il faudra donc avoir une grande discipline pour ne pas voir dans ce que nous appelons intestin, l'organe du même nom que la philosophie a soumis au cerveau.

Notre concept d'intestin n'est pas un organe : s'il est un corps, il est un corps sans organes. Il n'est pas, pour autant, une métaphore. Une métaphore est une façon de dire quelque chose autrement, avec un autre mot qui, renvoyant au même concept, montre qu'un même signifié peut se retrouver qualifié de deux signifiants différents que la métaphore, justement, relie presque magiquement. Or, par ce mécanisme, c'est tout le jeu du dualisme philosophique qui s'opère: une métaphore sous-entend qu'il existe nécessairement, derrière chaque signifiant (chaque mot), un signifié (un concept) qui ne serait accessible que par ce sésame du signifiant. C'est un peu comme s'il fallait 
nécessairement imaginer un intestin qui, dans les aliments qu'il ingurgite, digère le sucre nécessaire au fonctionnement du cerveau. La métaphore n'est que la forme la plus subtile, peut-être la plus sublime dirait Lacan, de ce dualisme que nous traquons. Il serait donc erroné de penser que nous construisons un concept comme métaphore du Capitalisme (qui digère tout), de l'écologie (le nucléaire comme merde de l'humanité en décomposition), de la consommation (la croissance comme nourriture infinie d'un intestin surpuissant), de l'urbanisme (la ville-tuyaux qui amène aux citadins l'eau à boire directement dans sa bouche-robinet et récupère ses excréments directement par son anus-WC), de tant de choses que l'on pourrait résumer à : 1-absorbtion, 2-digestion, 3incorporation et 4-excrétion - les fonctions de l'organe intestin.

Derrière le mot Capitalisme, le mot écologie, le mot urbanisme, le mot consommation, il n'y a pas un super-concept dont le super-signifiant (le mana des anthropologues, l'objet a des psychanalystes qui sont devenus des succédanés transcendantaux) serait intestin - les métaphysiciens ne savent faire qu'avec les métaphores. C'est pourquoi nous affirmons que l'intestin n'est pas une métaphore. Dans ce corps sans organes qu'est l'intestin, il n'y a pas de temps 1-2-3-4 - de temps chronologique. L'intestin resterait une métaphore dans un champ philosophique dualiste où il se soumettrait au temps (historique) du cerveau. Mais nous voulons voir dans l'intestin une sorte de temps pré-historique (la physique sans métaphysique), ou plutôt an-historique (ou, mieux : anti-historique) où toute métaphore perd son sens. Si intestin est autre chose que, dans le langage courant - qu'il soit japonais ou français -, celui des deux organes distincts que l'on nomme, justement pour les distinguer, cerveau (nô) et intestin tube digestif (chô), et si le lecteur veut savoir ce qu'il est, alors la meilleure réponse que nous pouvons lui procurer est qu'il est cerveau (nô) et intestin (chô), sans être l'un ou l'autre, tout en étant différencié quand même. Notre concept d'intestin? C'est notre livre à venir.

11 Remarque: nous dénommons donc, pour plus de clarté, l'intestin rabattu vers l'arrière " tube digestif ", et l'intestin plan de consistance, qui titre notre philosophie, simplement « intestin ».

\section{(I). Extensions de l'intestin}

Il y a donc beaucoup de vivant et de non-vivant, il y a beaucoup de machinique dans l'intestin. Dit d'une façon plus simple (et aussi plus ésotérique) encore, il y a de l'intestin dans l'intestin, et de l'intestin dans l'intestin de l'intestin. Un complexe matériel qui se comprend lui-même, c'est ainsi que nous comprenons l'intestin. Mais si l'intestin, comme une sorte de puits sans fond, se prolonge à l'infini au-dedans de lui-même, il n'a de cesse de s'étendre au dehors, faisant feu de tout bois, vivant ou non-vivant, microscopique ou cosmologique. S'il se laisse prendre à une définition - ce que nous avons la faiblesse de croire en écrivant cette introduction, même si nous savons qu'elle ne cesse de nous échapper - celle-ci répond à une organisation (et non pas: organisme) complexe connectant l'intestin infinitésimal et l'intestin infiniment grand. S'il a un nom, l'intestin s'appelle Gulliver.

\section{Extensions vers l'avant}

Prenons un exemple de ce que nous entendons par extension vers l'avant de l'intestin : les dents peuvent être considérées comme la part de l'intestin qui coupe, qui tranche le temps et l'espace. En effet, croquer ou mâcher, c'est du temps et de l'espace gagné pour 
l'intestin : la nourriture préparée en amont du tube digestif accélère le travail de celui-ci (il modifie le temps), et diminue les surfaces à digérer (il modifie l'espace de même). Les organismes qui sont pourvues de dents voient donc, avec leur intestin qui se prolonge, qui s'étend vers l'avant, le temps et l'espace se modifier et se diviser. Mais ces modifications ne sont pas que relatives à l'organisme qui les vit: cette extension de l'intestin modifie, en soi, le temps et l'espace : se crée alors un espace-temps extérieur, d'avant la coupure et la prédigestion par les modules d'extension de l'intestin (dont les dents), et un espace/temps intérieur, interne au processus digestif en tant que tel, qui est celui de ce qu'on appelle dans le langage courant l'intestin (le tube digestif, l'organe à l'intérieur du corps). La bactérie au contraire, qui n'a pas de dents (et que l'on suppose donc pour la commodité de la démonstration ne pas avoir d'extension de l'intestin, ce que nous pensons en réalité bien sûr impossible), a le même univers temporo-spatial au dedans et au dehors d'elle. C'est à ne pas pouvoir mâcher, par défaut de dents, que le serpent du petit prince se retrouve paralysé par un éléphant qui le transforme en chapeau géant dans un intestin infiniment extensible : l'espace et le temps, qu'ils soient envisagés du dedans ou du dehors de l'intestin, sont identiques. Un tube digestif qui ne posséderait pas cette capacité d'extension (nous répétons que cela est, pour nous, impossible) pourrait prendre n'importe quel forme, se soumettre au temps ou à l'espace extérieur, tout comme il imprimerait sa temporalité interne et sans fin à l'univers qui fait de même avec lui.

\section{Histoires naturelles : serpent, chat, thon...}

Ces exemples sont caricaturaux, et il ne faut pas les prendre au pied de la lettre, mais plutôt comme des images nous permettant de dessiner à grands traits, pour commencer, la figure de ce que l'on nomme habituellement l'intestin (chô) : en effet, si les serpents ne possèdent pas de dents au sens où les mammifères en ont, ils ont des crocs qui diffusent, grâce aux glandes qui leur sont attachées, des poisons qui préparent déjà la proie à la digestion; de même, certaines bactéries diffusent dans le milieu extérieur à leur cellulescorps des substances, voire même de prolongements sous forme de pseudopodes, qui sont aussi ce que nous appelons extension vers l'avant de l'intestin. Précisons donc, avant de prendre un troisième exemple tout aussi arbitraire, qu'il n'y a pas pour nous d'histoire naturelle au sens évolutif du terme (qui progresserait de la bactérie au serpent puis au chat, puisque le félin sera l'exemple suivant) mais plutôt une histoire naturelle au sens descriptif, presque naïf de découverte du monde : Pline l'Ancien plutôt que Buffon.

Mais revenons à notre troisième exemple d'extension vers l'avant de l'intestin : différent de la bactérie ou du serpent est le chat : l'intestin des chats, qui possède des dents (crocs et griffes contre intelligence et civilisation disait Schopenhauer), étend son monde bien au-delà des frontières extérieures de sa féline gueule. Au pro rata de sa taille, le chat, nous dit l'anatomie, possède un tube digestif bien plus grand que celui du serpent. Si le serpent a un court tube digestif (à peu près $28 \%$ de la longueur de son corps trouve-t-on sur wikipedia), celui du chat, qui mesure $1 \mathrm{~m}$ pour l'intestin grêle et 20 à $40 \mathrm{~cm}$ pour le gros intestin, outre sa différence de taille, fonctionne tout autrement. Les extensions de l'intestin que sont les dents du chat ne mastiquent pas mais coupent les proies : il ne peut en effet faire rentrer que de tous petits morceaux dans son tube digestif et est donc obligé de faire plusieurs repas par jour (une grosse dizaine), pour un transit global de 12 à 14 heures. 
16 On pourra nous rétorquer que si l'on se lance dans d'aussi techniques et complexes calculs, en divisant la quantité d'alimentation par le temps mis pour la digérer et en comparant la taille respective de chaque animal, le résultat reviendra peut-être au même pour le chat et pour le serpent. Mais ce serait ignorer que chats et serpents ne vivent pas dans le même monde. Le chat, au contraire du serpent, peut encore se mouvoir pendant qu'il est en train de digérer. Son appareil locomoteur, que nous considérons également comme prolongement, extension vers l'avant, de l'intestin, l'amène à chasser d'autres proies sans qu'il ait à arrêter sa digestion. Le serpent, lui, reste statique à attendre que l'éléphant ait été totalement ingéré - et c'est pourquoi Saint Exupéry a tout le temps pour l'imaginer comme un chapeau. Il arrive même, dit-on, qu'un serpent dérangé pendant sa digestion, dégurgite l'animal en partie digéré qu'il était en train d'absorber. Le chat trace à même son territoire de chasse les marques par son urine, par les empreintes de ses pattes, et celles de sa queue qui peut servir à effacer certaines des précédentes. Toutes ces marques sur la terre, toutes ces traces, sont encore de l'intestin - l'éducateur Fernand Deligny, quand il travaille avec les autistes, les appelle des lignes d'erre.

Nous considérons donc le territoire de chasse du chat comme de l'extension vers l'avant de l'intestin dont il faut, dans le calcul que l'on nous opposerait, tenir compte: la balance longueur du tube digestif / temps de digestion change alors considérablement si on inclut dans le calcul de la fonction intestinale (nous préférons ce terme à celui de «fonction digestive » qui n'en est qu'une limitation) cette dimension territoriale de l'intestin. Tout territoire sur lequel le chat se retrouve pourra donc, potentiellement, être intégré comme de l'intestin et permettre son extension. C'est parce que nous considérons que le territoire de chasse du chat, au fur et à mesure de sa rencontre avec lui, devient véritablement de l'intestin du chat, que nous nous permettons d'affirmer que le serpent et le chat ne vivent pas dans le même monde. Le philosophe Félix Guattari, lorsqu'il parle de la subjectivité de l'oiseau, ne l'arrête pas à son corps emplumé posé sur la branche d'un arbre à côté de son nid, mais l'étend, la baptisant ritournelle, à tout le champ aérien qui porte son chant : le chant du rossignol insomniaque, dont on ne sait où il se trouve en cette douce nuit d'été, c'est le rossignol lui-même qui vient nicher à notre oreille, en nous même. L'extension vers l'avant de l'intestin prend, avec les exemples du chat et de l'oiseau, une dimension plus vertigineuse encore.

\section{Omote (表) et Ura (裏)}

18 Une remarque avant de continuer cette histoire naturelle : cette extension, le lecteur aura remarqué que nous l'appelons «vers l'avant». Pourquoi? Parce que nous la différencierons, plus loin dans cette introduction, d'une extension vers l'arrière de l'intestin. En Japonais, s'opposent deux mots, qui décrivent tout autant l'intérieur (la partie cachée) que l'extérieur (la partie visible, apparente), le dedans que le dehors d'un vêtement, mais aussi le recto et le verso d'une pièce, l'avant et l'arrière d'un objet : omote (表) et ura (裏) - l'expression hyôri (表裏) signifie double face, recto verso. C'est en s'inspirant de la polyvocité de ces mots japonais que nous avons expliqué le côté avant (c'est-à-dire recto, partie extérieure, visible, apparente) de l'extension de l'intestin, tout comme nous développerons plus loin le côté arrière (verso, partie intérieure, invisible, cachée).

Après les chats, les oiseaux, il est tout naturel de s'intéresser aux poissons. Si l'on sait que le thon nage vite ( $150 \mathrm{~km} /$ heure), on imagine aisément pourquoi nous pensons l'océan 
comme l'intestin potentiel du thon, celui où il va, potentiellement, étendre son intestin : le territoire de chasse du thon est infiniment plus grand et complexe que celui du chat, car les courants marins, et le nomadisme des proies du thon sont d'un tout autre ordre que les caractéristiques des proies du chat (notamment plus sédentaires). D'ici à dire que les dents (crocs) du félin jouent le même rôle que les longs parcours annuels du thon, c'est néanmoins une affirmation que nous ne ferons pas, car si notre petite histoire naturelle se veut descriptive et non progressiste, elle ne saurait néanmoins en aucun cas être comparatiste. Mais s'il y a un point commun au thon et au chat, c'est qu'aucun thon n'a jamais réussi à parcourir la totalité de tout l'espace des océans de la terre, et qu'aucun chat n'a jamais arpenté dans sa totalité tout son territoire - ne serait-ce que parce que ces territoires, marins ou terrestres, outre leur vastitude, se modifient constamment. C'est pourquoi nous différencions, dans ce que nous nommons extension vers l'omote, vers l'avant de l'intestin, ce que nous appellerons, avec le philosophe Gilles Deleuze, l'intestin réel et l' intestin virtuel.

\section{Intestin potentiel}

Expliquons-nous. Il existe une relation mathématique entre l'intestin réel du chat et du thon (pas seulement leur intestin intracorporels - le tube digestif évidemment, mais aussi ses crocs et ses pattes pour le chat, ses nageoires et son instinct grégaire pour le thon mais aussi les chemin parcourus dans leurs territoires respectifs) et leur intestin potentiel, virtuel dirons-nous plus justement (le territoire de chasse terrestre pour le chat et océanique pour le thon) : les corps du chat et du thon, comme point d'entrée des aliments dans leur intestin intérieur sont les points différentiels entre l'intestin réel (le corps ou le territoire qui bouge avec l'animal - nous pouvons l'appeler, cet intestin réel, ritournelle: $c f$ le chant de l'oiseau de Guattari -) et l'intestin virtuel (qui correspond au point de jonction de l'animal avec le territoire et qui, potentiellement, est intégrable comme intestin réel).

21 Tout cela sera plus clair encore avec un exemple simple : imaginons l'oiseau, son corps avec ses plumes, sur sa branche, à côté de son nid, comme le point différentiel entre son intestin réel et son intestin virtuel. Son intestin réel ce n'est pas uniquement son tube digestif avec lequel il digère les insectes qu'il attrape, grâce à ses ailes et à son bec, mais aussi (extension vers l'omote, extension vers l'avant de l'intestin), c'est aussi son chant qui est entendu par un autre oiseau et qui va lui signifier de ne pas rentrer sur son territoire ou au contraire de venir y faire l'amour, ce même chant qui sera entendu par un poète et qui lui fera composer un magnifique quatrain. L'intestin virtuel de cet oiseau, c'est tout l'espace et tout le temps qui pourront devenir de l'intestin réel : par exemple le même poète qui ne faisait pas attention à l'oiseau car il était pris dans son livre et qui, tout d'un coup, l'entend et, par là même, est pris dans la ritournelles de l'oiseau; de même le papillon qui, alors qu'il était en dehors du champ de vision de l'oiseau, y pénètre pour son plus grand malheur. Autrement dit, l'intestin ne cesse, à ce point différentiel - terme à entendre au sens où une différenciation de fonction s'opère: le virtuel permettant le passage d'un réel à un autre; il ne s'agit pas forcément du corps individualisé de l'organisme qui possède le tube digestif -, l'intestin, donc, ne cesse à ce point différentiel d'avoir la possibilité de s'étendre toujours plus encore vers l'avant. 


\section{Prométhée et le don du feu comme prototype de l'extension vers l'avant de l'intestin}

Prenons à présent le rôle du feu pour l'humanité : donné par Prométhée, il est la preuve de cette humanité. Il constitue, pensons-nous, un prolongement de l'intestin infiniment plus considérable que les dents : si le feu réduit l'espace et le temps, il le fait plus avant encore que les dents. Les aliments cuits prolongent l'intestin plus avant encore que ne le font les dents: les aliments cuits sont évidemment plus facilement assimilables par le tube digestif ce qui représente un changement radical du temps et de l'espace. Si les méandres du trajet océanique sont les dents du thon, si les crocs qui marquent son territoire sont les dents du chat, le feu peut être considéré comme les dents de l'humanité. Mais cela ne suffit pas: dents de dents de dents, l'humanité ne sait pas faire autre chose que de laisser se prolonger, plus devant et plus arrière, l'intestin - le lecteur comprendra alors peut-être mieux encore pourquoi nous avons gardé, pour parler du plan de consistance, du corps sans organe, de ce chant des entrailles que nous traquons, au-delà de tout dualisme, le concept d'intestin. Une fois l'intestin devenu feu, le genre humain se met à brûler (cuire) d'autres choses que ses aliments : du fer fondu il fait des fourchettes et des couteaux. Et pour couper les arbres d'où il extraira les baguettes qui servent pour d'autres humains de fourchettes et de couteaux, il fabrique des haches de métal. Le feu, comme dents, se prolonge(nt) de façon matérielle et immatérielle, par des objets tout autant que par des concepts, armes pour se défendre et attaquer, électricité et ordinateur pour rationnaliser, livres et temples pour donner du sens, etc. - la philosophie occidentale comme le point différentiel où l'intestin, le chô passe au service du cerveau, du nô... mais l'on va y revenir.

Le feu, capable de tout brûler, est le point différentiel qui permet à l'humanité de faire de tout intestin virtuel un intestin réel : d'une forêt luxuriante un champ de blé ; d'un atome d'uranium, un territoire irradié. Qu'apportons-nous d'original dans tout cela? La connexion : nous ne voyons pas de solution de continuité entre une humanité placée d'un côté, culturelle, et un feu naturel placé de l'autre, dont le pont jeté serait Prométhée. Quand nous disons que le feu est les dents de l'humanité, il ne s'agit pas d'une métaphore dont Prométhée serait le symbole: pour nous, le feu participe de l'homme comme l'homme participe du feu. Autrement dit, il n'y a pas d'un côté un cerveau (les dieux donnant le feu, comme concept, aux hommes) et de l'autre un intestin (les hommes recevant, dans leur corps, le cadeau des dieux) et, comme médiateur, Prométhée : il y a seulement un intestin qui porte comme nom Prométhée. S'il existe des prolongements biologiques, vers l'avant (dents, territoires de chasse, etc.) de l'intestin humain, celui-ci fait le choix de se prolonger, de façon non organique, par le feu, et de soumettre tous ces points différentiels qu'il croit tenir des animaux, au feu. Autrement dit, avec nos mots à nous, Prométhée a appris à l'homme à faire du feu le point différentiel qui lui permet de prolonger son intestin. D'une certaine manière, l'humanité a fait en sorte, grâce à Prométhée, de réduire le point différentiel qui lui permet de digérer tout ce qui se présente à lui, à un et un seul mécanisme : le feu.

Alors, bien entendu, ce mécanisme prend une infinité de formes : mais entre la tragédie de Fukushima, celle de Tchernobyl, la nécessité pour des paysans sud-américains pressés par des compagnies multinationales à faire de la monoculture à grand renfort d'engrais de détruire la forêt amazonienne, entre les repas tout fait, transgéniques, qui inondent les 
micro-ondes des foyers du nord et le mil pillé de façon ancestral en Afrique et cuit pour faire des crêpes riches en glucides et pauvres en lipides, entre un Nietzsche qui découvre l'éternel retour en Europe au XIX siècle et un Bashô qui s'émerveille devant le temps éternel du voyageur au XVII ${ }^{\mathrm{e}}$ siècle au Japon, entre toutes ces extensions de l'intestin humain, le point différentiel que Prométhée nous a appris, à l'exclusion de tout autre, c'est le feu. C'est pour cela que l'intestin devient la machine du feu : le feu se transmet à tous les niveau de la digestion intestinale : l'intestin mange le feu qu'il brûle et qu'il expulse, qu'il crache ou qu'il chie. Prométhée n'a pas donné le feu à l'humanité, il lui a appris à digérer le feu, à le manger, le cracher, le chier : Prométhée a fait de l'homme un dragon (le dragon scandinave, des volcans, pas le dragon japonais, aquatique), il en a fait une salamandre.

Le problème est que, à sa suite, et en son nom, l'intestin a été récupéré par une tradition philosophique qui a mis de la dualité là où il n'y avait que prolongement infini. Le feu a été le point d'entrée du dualisme - comme dans la légende japonaise où la mère du monde, Izanami, crée la distinction entre la mort et la vie en donnant naissance au feu son fils. Le dragon du feu, d'intestin s'étendant sans cesse vers l'avant et vers l'arrière, s'est rabattu sur un tube digestif (appelé intestin), une énorme structure intestinale coiffée d'un chef terrifiant : le cerveau.

\section{(II). Par-delà intestin et cerveau, chô et nô, avant et arrière, omote et ura}

Est-ce à dire que le cerveau est une évolution ultime de l'intestin ? Oui et non : oui, car il correspond à ce processus d'extension vers l'avant de ce que nous avons défini comme l'intestin. Et non car, nous l'avons dit, il n'y a pas de solution de continuité, de hiatus entre le cerveau (nô) qui serait avant (au dessus de) l'intestin (chô). Pour nous, il n'existe qu'une entité : les entrailles que nous appelons intestin. Le problème philosophique étant que, pour des raisons qu'il nous faudra élucider, le chô s'est différencié du cerveau nô, soumettant celui-ci à celui-là.

Toute structure est aussi le prolongement de l'intestin, que nous disons vers l'avant, à travers le cerveau, en utilisant le cerveau: structurer une recette de cuisine permet de gagner du temps, et de même la cuisine (comme espace) sera d'autant plus efficace qu'elle sera structurée. Ainsi, au bout de la chaîne de production alimentaire, la gestion de la nourriture se réduit et s'organise au strict minimum: ex.: une barquette de hachis Parmentier préparée industriellement et à mettre au micro-onde (seul appareil irréductible dans la cuisine). Mais cela étend nécessairement le temps et l'espace, en amont, de production desdits aliments: abattoir, usine d'emballage, transport, production, distribution, fabrication de la machine, des ondes, de l'électricité, du gaz, des trains, des usines de retraitement - ce que nous appelons dans le langage courant l' industrie... La cuisine (le lieu résumé à la cuisine) et la recette (mettre la barquette dans le micro-onde) sont réduit à l'infiniment petit et, par contre, tout le réseau de fabrication en amont est étendu à l'infiniment grand.

Nous identifions la structure de la cuisine (recette + lieu), cette concentration, condensation, au cerveau : non pas pace que toute cette organisation (la construction et l'exécution de la recette minimum qui consiste à mettre la barquette dans le micro-onde) nécessite un cerveau - une moelle épinière suffit pour mettre une barquette de hachis 
Parmentier dans le micro-onde - mais nous faisons cette identification parce que le cerveau peut se résumer au minimum, au plus fin : à un neurone. En effet, le cerveau estil autre chose qu'une membrane qui laisse passer ou non des informations? La synapse qui code les informations électriques en 1 ou 0 et qui ne fait que gérer celles qu'il peut comprendre, fait exactement comme l'intestin qui, lui aussi, est une membrane qui laisse ou non passer les aliments qu'il peut digérer. Le cerveau peut tout à fait se résumer à une barrière d'échange aussi fine qu'une membrane dont le fonctionnement est très simple : un élément en amont de la membrane passe ou ne passe pas, à travers cette membrane, en aval.

Une membrane n'a pas besoin de beaucoup d'énergie pour vivre : on sait que le cerveau ne se nourrit que de molécules de sucre, ce qui veut dire que seules les molécules de sucre lui suffisent. Comme dans un roman de science fiction (captain Future, héros de pulps américain, développé à partir des idées de l'écrivain Edmond Moore Hamilton dans les années cinquante, et célèbre en France pour son adaptation en manga nommé Capitaine Flam) où un des personnage qui s'appelle le Pr Simon Wright, après avoir vécu une vie «normale » (avec un corps, et donc un tube digestif), se résume à son cerveau placé dans une bulle remplie d'eau et de solution sucrée : totalement autonome, il n'a besoin que d'esclaves qui fabriquent pour lui, à l'extérieur, la substance indispensable (le sucre) au cerveau qui les dirige. L'intestin est, dans cette fantaisie de science fiction, devenu totalement extérieur au cerveau (et même à son corps), montrant ainsi que si cerveau et intestin ne cessent, suivant les tendances philosophiques ou idéalistes (l'intestin soumis au cerveau) ou matérialiste (le cerveau soumis à l'intestin), de jouer à qui dominera l'autre (le jeu, à la Hegel, du master and servant), ils semblent visiblement être, malgré tout, assez indépendant l'un de l'autre. D'où notre question : pourquoi les associe-t-on systématiquement, le plus souvent pour inféoder l'intestin au cerveau?

\section{Embryologie}

L'embryologie pourrait sembler être d'une grande aide pour comprendre les liens physiologiques entre le cerveau et l'intestin. En effet, l'embryologie nous apprend que le tube digestif et le cerveau se développent de façon parallèle, sans être aucunement en lien l'un avec l'autre. Quand le spermatozoïde rencontre l'ovule et crée l'œuf qui deviendra l'embryon puis le petit d'homme, il développe, à la deuxième semaine de développement, deux tissus différents et hétérogènes nommés ectoblaste et endoblaste. Ces deux tissus donneront, respectivement, les cellules nerveuses (cerveau et système nerveux périphérique) et les cellules de la peau et de l'appareil intestinal. Ainsi, d'un point de vue ontogénétique (développement de l'individu), le cerveau et le tube digestif sont deux mondes qui se développent en même temps mais en parallèle : l'embryologie nous montre bien que le cerveau ne descend pas de l'intestin, comme organe, et que celui-ci ne descend pas non plus du cerveau.

Nous avons, plus haut, cité le travail des physiologistes sur les hydres: pour eux, nous l'avons dit, les cellules nerveuses sont un développement, au cours des millions d'années d'évolution terrestre, des cellules intestinales (les paraneurones). Mais cette version phylogénétique (la phylogénèse s'occupe du développement de l'espèce) ne nous est ici d'aucune aide : nous avons déjà montré qu'elle pouvait nous induire en erreur et inverser la métaphysique (qui soumet le tube digestif (chô) au cerveau (nô) en inféodant le cerveau (nô) à la toute puissance de l'intestin comme tube digestif (chô). Nous nous contenterons 
de dire que le cerveau et tube digestif, d'un point de vue physiologiques, sont hétérogènes et irréductibles l'un à l'autre. S'ils sont mis en lien dans un rapport hiérarchique c'est uniquement à la (mauvaise) philosophie qu'on le doit - et c'est celle-là que nous voulons démonter.

Quoi qu'il en soit, au delà de cette dualité que nous voyons en fait beaucoup horizontale (développent parallèle) que verticale (fausse hiérarchie due à une fausse philosophie), cerveau et tube digestif se rejoignent, comme les droites parallèles à l'infini, au sein du véritable intestin, celui qu'avec Guattari-Deleuze nous nommons plan de consistance : cet intestin sort du corps, il est tout autant à l'extérieur qu'à l'intérieur du corps : s'il a encore à voir avec le corps, il ne résume pas à lui : il s'étend à l'infini, devant et derrière, vers le futur et dans le passé, dans tout les sens, pour tout absorber : il est le monde (le monde comme corps). Le cerveau ne préexiste pas à le tube digestif pas plus que le tube digestif ne préexiste au cerveau : ce n'est pas plus le cerveau qui utilise le tube digestif que le tube digestif qui utilise le cerveau. En fait, l'intestin, se prolonge chez l'homme grâce au don de Prométhée : le feu - vers l'avant en cerveau et vers l'arrière en intestin (tube digestif) : mais, au bout du compte, comme l'ura et l'omote, il n'en reste pas moins celui que nous nommons l'intestin, un plan de consistance.

\section{Extension vers l'arrière}

Cette prolongation vers le monde et le cerveau, nous la disons donc vers l'avant. Mais l'intestin se prolonge aussi vers l'arrière: c'est ce que nous avons l'habitude d'appeler l'intestin et qu'il faudrait appeler, pour le distinguer du plan de consistance, le tube digestif, celui qui va du réfrigérateur et du robinet vers les W.C., de la bouche à l'anus. Quand nous parlons du tube digestif, ce ne sont pas vraiment les aliments qui pénètrent dans la bouche et sortent par l'anus qui nous intéressent, ce n'est pas le mécanisme que l'on nomme couramment digestion que nous voulons analyser. Nous avons réussi à comprendre que l'intestin s'étendait vers l'avant, englobant le monde tout entier, par une transformation en cerveau. C'est par le mécanisme de digestion du feu (Prométhée) que nous avons pu le comprendre : le feu, avons-nous dit, est le point différentiel où l'intestin virtuel devient réel (dans le cas de l'homme, il se réalise en dualisant le corps en cerveau et en tube digestif). Pour comprendre ce que nous appelons, par commodité, l'extension vers l'arrière de l'intestin (qui participe de la même dualité), c'est-à-dire le mécanisme de création du tube digestif, nous pouvons prendre un autre élément que le feu : l'eau.

Pourquoi ces deux éléments? A cause de leur complémentarité - et de leur différence : ce n'est pas seulement parce que Hippocrate, et Galien à sa suite, résumaient le monde en quatre élément opposables deux par deux (terre/air; feu/eau), comme binômes dynamique et statique, positifs et négatifs, que nous les suivons dans cette alchimie en utilisant le feu comme modèle de l'extension vers l'avant de l'intestin (le cerveau, nô) - les bûchers de la Renaissance, feux de l'extermination des sciences intestinales de l'herboristerie des sorcières et des plaisirs intestinaux des sodomites qui terrifiaient tant les universitaires scholastico-sorbonnards - et l'eau comme modèle de l'extension vers l'arrière de l'intestin (le tube digestif, chô). C'est aussi parce que l'eau et le feu sont les deux éléments qui empêchent les Japonais et les Français de se comprendre : si on évoque à un Japonais la figure du dragon, il pensera à l'eau; si on évoque la figure du dragon au Français, il pensera au feu. L'eau et le feu sont ce qui les empêche de se comprendre et ce qui, pourtant, les réunit, car de l'extrême orient à l'extrême occident, ces deux hommes 
pensent au même dragon, qui pourrait être un autre nom de l'intestin - du plan de consistance.

Mais ne nous y trompons pas : l'eau et le feu ne sont pas les métaphores du cerveau et du tube digestif, nouveau rabattement dualiste de l'intestin. L'eau et le feu sont dans un rapport beaucoup plus étroit, beaucoup plus intime, à nouveau comme l'ura et l'omote de l'intestin. Gabriel Tarde peut nous aider à conceptualiser le feu et l'eau: le feu est un point différentiel avons-nous dit. Qu'est-ce que cela signifie quant à l'intestin? Le feu serait comme une sorte d'irruption du réel au sein du virtuel (irruption qu'on pourrait dire aussi du virtuel au sein du réel), un point d'incandescence où apparaît, fiat lux, quelque chose, une production de l'intestin (de la naissance de la vie à sa disparition). Le feu correspond, pour utiliser les termes de Tarde, à l'invention, qui sort des ténèbres chaotiques pour s'élever, presque de façon immatérielle, vers les lumières aériennes. Il s'agit donc bien d'un processus d'extension, mais d'une extension en point, en ligne, concentrant un maximum d'énergie en un minimum de temps et d'espace (une sorte de naissance d'une étoile ou de fin de celle-ci).

L'eau, au contraire, s'écoule, possède cette tendance extraordinaire à épouser toutes les surfaces avec lesquelles elle est en contact. Si quelque chose nait de l'eau, c'est toujours sous la forme d'une ondulation, que celle-ci soit concentrique et centrifuge comme lorsque l'on jette un caillou à sa surface, ou qu'elle soit massive comme les vagues de l'océan. L'eau est également un processus d'extension - Tarde la dit processus d'imitation. Par imitation il faut comprendre la reproduction d'un mouvement original qui se transmet, comme dans le jeu de dominos, d'une molécule d'eau à une autre molécule d'eau: ici, l'extension n'est pas concentrée mais au contraire dilatée, en surface, dispersant un maximum d'énergie en un maximum de temps et d'espace (l'extension de l'univers après sa naissance ou avant sa mort).

Nous n'expliquons pas ici ces deux mécanismes pour les opposer. Lorsque l'intestin s'étend, c'est-à-dire qu'il produit quelque chose de nouveau, il le fait toujours vers l'avant et vers l'arrière : il le fait par le cerveau et par le tube digestif : il développe en son sein le feu et l'eau. Comme nous avons déjà dit plus haut, utilisant les images du ciel et de la terre, de l'homme et de la femme, de l'enfant et du parent, l'intestin s'étend toujours du plus qu'il peut, et c'est l'erreur de la métaphysique d'opposer, de dualiser, ces mécanismes d'extension qui ne sont en fait qu'une seule et même réalité.

\section{La ville comme prototype de l'extension vers l'arrière de l'intestin}

L'extension vers l'arrière de l'intestin a donc à voir avec l'eau. L'eau est le point différentiel - rappelons qu'un point différentiel est pour nous le passage de l'intestin virtuel à l'intestin réel -, l'eau est donc un point différentiel qui fait qu'un corps absorbé par le tube digestif va être digéré, disséqué, traité, étendu à l'infini par ce tube digestif pour que chaque molécule qui le constitue soit ensuite intégrée par ledit tube. L'eau est le liant des corps (tout le monde sait que, sans eau, tout corps se résume à un tas de cendres, presque comme s'il était le reste d'un incendie), et c'est par la déliaison avec l'eau, par les jeux de "désaquification» et de "réaquification» (termes plus précis que dé- ou réhydratation) des corps que le tube digestif joue son rôle dans l'organisme : la digestion. (Précisons que nous englobons dans le tube digestif le système rénal qui traite les déchets liquides pour les éliminer en urine : ce que le rectum et l'anus sont pour les solides, la vessie et le méat urinaire le sont pour les liquides). 
39 Des solides ou des liquides, ces systèmes d'évacuation au sein du corps nous évoquent quelque chose comme un paradigme des échanges sociaux. Il semble en effet qu'une société qui se civilise s'organise essentiellement autour de la gestion de ses propres déchets corporels (avant même de traiter ceux qui sont issus de sa consommation) - à l'origine du film Dune de David Lynch, le rôle de l'Empereur devait être tenu par Salvador Dali qui avait imaginé un trône en forme de toilettes, avec deux orifices séparés car « rien n'est plus vulgaire que de mélanger le caca et le pipi ». Un regroupement humain, pour vivre, se doit d'organiser la façon dont il va évacuer, éliminer, rejeter ses excréments et ses urines. Une ville, disons-nous, n'est rien d'autre que l'organisation complexe d'un ensemble d'individus humains qui s'organisent pour ne pas vivre au milieu de leurs déjections. Imaginons un groupe d'une dizaine d'individus vivant à la campagne : lorsque l'un d'entre eux veut éliminer ses selles ou ses urines, et qu'il ne peut pas, pour des raisons de conventions sociales (religieuses, hygiéniques...) le faire au milieu de ses congénères, il lui est facile d'aller à l'extérieur de l'habitat qui les héberge, dans un lieu réservé à cet usage. Cela est évidemment impossible dans une ville où il habite au centre, comme Paris si l'individu en question habite Oberkampf ou Tôkyô s'il habite Shinjuku. Pour aller à l'extérieur de la ville qui le contient ainsi que ses congénères, il lui faudrait parcourir un nombre conséquent de kilomètres que son tube digestif ne supporterait certes pas avant que de se vider - un sociologie plus excitante que celle qui nous est servie quotidiennement, aurait tout intérêt à compter le nombre de toilettes publiques à Paris et à Tokyo...

40 Ainsi, avant tout, un regroupement humain se doit de penser, pour permettre à chacun des humains de faire ce qu'il a à faire au milieu du grand nombre (travailler, circuler, consommer, produire...), la façon la plus efficiente, la plus adaptée, de gérer le problème de l'excrétion des selles et des urines. Et c'est cette gestion qui donne naissance, pensonsnous, au concept de ville. On juge en effet une civilisation (de Machu Pichu à Rome, jusqu'à Londres, Paris, New York ou Tokyo, Rio de Janeiro ou Dehli) à sa capacité de gérer les eaux "propres » (en amont de leur utilisation) et les eaux " usées » (en aval de leur utilisation). Elle prend l'eau du ciel, des rivières et des mers pour la rendre potable et utilisable par le tube digestif de chacun de ses habitants, et restitue cette eau souillée dans un système de canalisation tout aussi compliqué qui ne gêneront aucunement chacun des habitants de cette ville. Autrement dit, la ville fonctionne, elle-même, comme un gigantesque tube digestif capable d'ingérer tout ce qui en dehors d'elle, de le digérer (point différentiel de l'eau: dissection et assimilation de ce qui lui semble utile, l'intestin virtuel devient réel), et enfin de l'éliminer. N'est-ce pas ce que les villes font des campagnes? Voire même les pays du Nord (comme concentration urbaine particulièrement organisée quand au traitement des déchets) vis-à-vis des pays du Sud si l'on nous oppose que les ville des pays dits du Sud sont aussi, voire plus, nombreuses et plus peuplées que les villes du nord, c'est que l'on aura pas compris notre raisonnement: il ne s'agit pas ici de caractériser une ville par sa taille ou sa population que par son organisation en termes d'égouts, de système de canalisations, et les villes du Sud, certes plus peuplées, le sont aussi par l'extension en bidonvilles où le système de retraitement des eaux usées n'est pas la préoccupation première des maires desdites villes. 


\section{Extensions vers l'avant et vers l'arrière de l'intestin : deux processus différents, mais complémentaires, qui expliquent les dualismes}

41 C'est pourquoi nous disons que l'intestin s'est prolongé vers l'arrière parce que, contrairement à la prolongation vers l'avant où l'homme devient un cerveau (un point différentiel concentré comme l'est le feu) qui, comme rabattement de l'intestin, prend le monde tout entier au piège de sa raison, rend métaphysique chaque chose, en distribuant ce qui est et ce qui n'est pas (et qui, donc, étant un n'étant pas, ne peut pas être). Dans le processus de prolongation vers l'arrière, l'homme se dissout dans la ville comme image du tube digestif (l'eau comme point différentiel l'étend du plus qu'il peut, comme l'étalement de l'ondulation à sa surface) qui rabat l'intestin en intestin civilisé, qui sait quoi manger, comment le déféquer, où le chier - il mange le reste du monde qu'il dit barbare, et lui chie dessus les résidus de sa digestion : la ville est un intestin primaire, une gigantesque organisme qui mange, par exemple, les pays du sud et les campagnes, et rejette ses excréments sur eux.

Mais vers l'avant et vers l'arrière, ces deux mécanismes sont en constante superposition. La ville prend les substances primaires mais aussi l'énergie qu'il faut digérer pour nourrir le processus, que nous disons vers l'avant, de création du cerveau - le sucre nécessaire à la survie du Pr Simon de Capitaine Flam (la consommation de sucre d'un pays est un bon repère de son quantum d'impérialisme). Et le cerveau en arrive à concevoir le monde uniquement comme ce qui est civilisé, laissant animaux, barbares et choses du côté du non-être, de l'absence de logos. Il lui faut bien, au cerveau, se justifier à lui-même que ce n'est pas sur du même qu'il chie, et que chier sur la tête d'une vache ou d'un Africain pauvre, ce n'est après tout pas si grave si ceux-ci ne sont pas vraiment comme lui et ses congénères, c'est-à-dire qu'ils n'ont pas le même cerveau ni le même tube digestif. C'est ainsi que le rabattement de l'intestin en cerveau et tube digestif rend chacun des deux éléments interdépendants l'un de l'autre : la physique et la métaphysique, l'une soumise l'autre et vice versa.

Mais, bien entendu, ce rabattement n'est pas sans poser soucis car, ainsi, existe un mouvement, qui ne saurait être éternel, où l'épuisement des ressources (amont) et le manque d'espace de rejet des déchets (aval) se creuse. Le cerveau se trouve en effet face à un problème de taille: il ne peut ingérer que ce qui est extérieur à lui et rejeter les résidus qu'à l'extérieur de son monde or, nécessairement, plus il grandit, plus son monde devient pléthorique, universel, plus il s'éloigne ce qu'il peut ingérer et de l'endroit où il va déverser ses déchets. C'est pourquoi le cerveau, refusant d'empiéter sur ses prérogatives d'extension, étendant toujours plus son monde, va voir son tube digestif grandir d'autant et apprendre dès lors à rejeter à l'extérieur de se monde ce qu'il y a prélevé. Et, s'étendant du plus qu'il peut, comme l'onde sur l'eau, le tube digestif pousse le cerveau à concentrer sa puissance, à se condenser en un point d'ignition maximum.

Un exemple montrer très bien ce mécanisme : c'est l'utilisation de l'atome par l'humanité moderne, à savoir 1) l'énergie d'une centrale nucléaire, où le feu se réduit (génie du cerveau d'Einstein), se condense en un noyau, et 2) où les résidus radioactifs de cette digestion - inondant des zones qui, même en son sein, sont considérés comme autres, comme toilettes (la Hague (France) et Rokkasho (Japon) - s'étendent de plus en plus à la surface du globe terrestre. De l'infiniment petit du cerveau à l'infiniment grand de l'intestin. Et si les pays du Sud et les campagnes refusent de se laisser digérer, ou si ils en 
viennent à s'offusquer que les villes du Nord leur chient dessus (s'incluant eux-mêmes, par la même occasion, à ce cerveau et à ce tube digestif urbain)? Qu'à cela ne tienne, la ville/tube digestif pourra toujours, comme certains «spécialistes » le proposent, rejeter ses produits contaminés radioactifs vers le soleil, lui-même alors conçu comme horsmonde, à côté des pays pauvres et des campagnes, tout ça devenant garde-manger et toilettes d'un intestin se prolongeant éternellement vers l'avant et vers l'arrière.

L'apparent paradoxe (c'est le livre à venir qui montrera l'apparence en question), c'est que pour construire un intestin-monde autrement appelé cerveau, le tube digestif s'organise en excluant de ce monde l'énergie qu'il absorbe et les déchets qu'il expulse. C'est pourquoi ce cerveau (occidental, du nord) croit être à lui seul le monde tout entier alors qu'il en a exclu, de ce monde, la plus grande partie (jusqu'au soleil même).

\section{(IV). Cannibalisme et coprophagie}

46 Nous pensons ici bien sûr au cannibalisme, et à son pendant, la coprophagie. Il existe une règle anthropologique, sur laquelle nous reviendrons bientôt, mais acceptons-la pour l'instant, qui veut que tout individu humain ne peut que considérer avec horreur le fait de manger un autre individu humain appartenant à son groupe humain. Ce qui ne veut pas dire qu'un homme ne puisse pas manger un autre homme, loin de là : il lui suffit de considérer cet homme, lorsqu'il le mange, comme n'appartenant pas à son groupe social, comme étant un autre. L'épisode traumatique du Radeau de la Méduse en est un exemple, où l'incompréhension éclate à la face de la civilisation occidentale naissante devant des individus qui préfèrent manger les leurs plutôt que de se laisser mourir de faim! Même les sauvages cannibales ne mangeaient que leurs ennemis, ceux qui n'appartenaient pas à leur groupe ! Quel constat amer pour les philosophes éclairés du siècle dit des Lumières.

Mais ne nous y trompons pas, cette règle est toujours d'actualité : et si l'anthropophagie (étymologiquement dérivé de anthropos, humain, l'anthropophagie ne concerne que le fait de manger des hommes), comme concept, a pris le pas sur le cannibalisme (étymologiquement dérivé du mot caniba utilisé par les Tainos rencontrés par Christophe Colomb et qui signifie, pour un individu - animal ou humain - manger un autre individu de son espèce), c'est que le groupe humain s'est élargi à toute l'humanité : du blanc au noir, du Japonais au Français, de l'acteur hollywoodien au trisomique, du vieillard au fotus... Aucun homme n'étant en dehors du groupe social d'un autre homme - l'esprit démocratique dicte que tout homme est forcément humain -, quiconque mangerait un autre que lui serait, de fait, anthropophage - c'est-à-dire cannibale humain. Quand nous disons manger un autre homme, cela inclut bien entendu tout autant le manger entièrement qu'en manger une partie - la partie pouvant être une production du corps de l'autre: le sperme (pratique sexuelle dite déviante), le sang (fascination pour les vampires) et, bien sûr la merde (coprophagie).

Le cerveau, comme extension vers l'avant, au point différentiel du feu, de l'intestin, se retrouve face au même problème: il ne peut se manger lui-même ou manger ce qu'il considère comme lui-même (y compris ses propres déchets). D'où la nécessité, s'il veut quand même le faire - ne serait-ce que pour ne pas mourir (comme les passagers du Radeau de la Méduse) ou pour continuer à croître - de faire en sorte que 1) tout ce qu'il absorbe ne soit pas lui, et que 2) ce qu'il excrète ne soit plus lui. Il doit donc se persuader que ce qu'il mange n'est pas encore lui et que ce qu'il élimine n'est plus du tout lui. L'extension vers l'arrière de l'intestin (tube digestif / point différentiel de l'eau) permet 
une telle chose en organisant un réseau d'excrétion de ses déchets si vaste qu'il puisse repousser le plus loin possible cette merde que le cerveau ne saurait voir (comme l'hypocrite Tartuffe de la pièce de Molière qui s'offusque devant le sein qu'il désire) jusqu'au soleil...

\section{Mythe de l'invention du foyer}

Il s'agit donc de penser ce qui est de l'ordre du commun et de l'autre, de l'intérieur d'une communauté et de son extériorité. Pour comprendre comment le cerveau, créé par et avec le feu avons-nous dit, en arrive à se considérer comme une communauté à lui tout seul, il nous faut réfléchir à la façon dont les communautés humaines ont vu le jour. Quant à nous, nous considérons que l'invention du feu et l'invention de la communauté sont une seule et même chose. Devenir maitre du feu, pour un groupe humain, doit passer par le fait de se trouver, collectivement, à manger à la même table. Précisons tout d'abord que nous pensons que pour domestiquer le feu (le récupérer d'un arbre qui brûle à cause de la foudre ou le créer en frottant deux morceaux de bois ou en frappant deux silex l'un contre l'autre), pour le maintenir en vie (dans un foyer), il faut plus qu'un homme, il en faut plusieurs. C'est pourquoi nous disons que l'invention du feu a à voir avec celle de la communauté, cette dernière étant nécessaire à l'acquisition de ce premier.

Pour étayer plus encore notre thèse, imaginons, un peu comme Vico, Hobbes ou Freud en leur temps, une sorte de mythe de fondation originel : lorsque les hommes vivaient seuls, individuellement, chacun d'eux étant différent de l'autre pouvait tout à fait dévorer un autre homme puisqu'il le considérait, de fait, comme différent de lui. De plus, tout préoccupé de sa survie individuelle, cet homme individualiste n'avait guère le temps de se poser la question de la nécessité et de l'utilité du feu : seul, il n'avait pas les moyens d'une telle ambition (l'ambition d'une extension vers l'avant de l'intestin en cerveau). Ainsi, les hommes, parce qu'ils sont, avant cette mise en commun, chacun individuellement différents les uns et des autres, peuvent donc se manger les uns les autres sans aucun problème : le cannibalisme, à cette époque charnière entre l'animal et l'homme, est, pour paraphraser Descartes, la chose la mieux partagée du monde.

Puis, pour tout un tas de raisons, dès que les hommes se rassemblent (nous n'entrerons pas ici dans les débats, par trop métaphysiques trouvons-nous, les disputes (scolastiques) entre Hobbes et Rousseau, Freud et Levi Strauss, de savoir pourquoi ils le font: plus grande efficace pour survivre, nécessité géopolitique, logique inhérente à l'espèce humaine, etc.), c'est-à-dire qu'ils se mettent ensemble autour d'une table - et donc d'un foyer (feu), d'une maison, d'un village, d'une ville -, en délimitant ainsi un intérieur (eux) et un extérieur (les autres), le cannibalisme se socialise et, d'universel, devient plus orienté: le cannibalisme des autres (ceux du groupe extérieur) devient socialement acceptable alors que celui des membres de son groupe devient interdit. De même, nous pensons que l'invention des toilettes suit le même chemin. Autrement dit, pour nous, l'invention des toilettes et du feu sont symétriques et probablement contemporaines: manger collectivement à la table commune et chier ensemble dans des toilettes communes est ce qui permet aux hommes de vivre ensemble et de se représenter comme un tube digestif commun. 


\section{Mythe de l'invention des toilettes}

52 Expliquons-nous : délimitant un intérieur et un extérieur (ce que nous avons vu que la création du foyer autour de la table commune permettait), et utilisant les toilettes pour expulser leurs déjections dans cet extérieur, on comprend que, comme pour le cannibalisme, les hommes de ce groupe ne puissent concevoir de manger leurs excréments au sein de leur propre groupe (à l'intérieur) alors que, retraités dans et par l'extérieur (dans la campagne alentours où ils deviennent de l'engrais alimentant la terre), ils pourront alors les manger puisque leurs déjections, devenus extérieurs, seront alors autres. Dès qu'une table et des toilettes deviennent communes, les hommes se fixent et délimitent une maison, puis une ville, avec un intérieur (eux, c'est-à-dire le même) et un extérieur (là où il n'y a pas la table ni les toilettes, c'est-à-dire l'autre). Ainsi, si la merde ne saurait rester à l'intérieur de la maison et de la ville (elle ne pourra être retraitée afin d'être consommée sous une autre forme, comme de l'autre), l'endroit où elle est excrétée doit nécessairement s'y trouver, à l'intérieur, puisqu'elle est encore, le temps de l'excrétion, une production du corps, du même (elle sera alors, dans cet intérieur, objet de répulsion, et l'idée de la manger une abomination).

Ceci explique l'étrangeté du fait que les toilettes, qui sont le lieu de sortie de ce qui dégoûte le plus les hommes, se trouve au sein de chacune des maisons du monde occidental. Un fait, connu au Japon, illustre cela admirablement : jusque dans les années 60 , dans les villages agricoles où les paysans vivaient de façon très individualisée et en autarcie, il était de coutume d'utiliser ses excréments directement dans son propre jardin, comme engrais (le régime alimentaire, végétarien et peu animal, bouddhisme oblige, et donc l'utilisation de toilettes sèches explique ce fait : les excréments des riches, dont le régime alimentaire était de meilleurs qualité, était d'ailleurs source de profit). Nous sommes ici face à une sorte de cannibalisme (sous forme de coprophagie), très différent du mécanisme de la ville qui nécessite un réseau complexe pour évacuer ses excréments loin d'elle (pour pouvoir les consommer une fois transformée dans cet extérieur, une fois devenus autres). On peut voir dans l'exemple de ces paysans japonais une sorte d'archaïsme du fonctionnement des individus avant le regroupement en ville.

\section{Le cannibalisme comme clef de voûte de la métaphysiques}

Une question reste bien entendu à présent en suspens - nous avons dit plus haut qu'il nous faudrait la traiter: pourquoi la civilisation humaine (au sens de l'extension du groupe social humain) a-t-elle aussi peur du cannibalisme, de se manger elle-même ? Une réponse simple (qui pourrait sembler, si on n'y prenait gare, simpliste) serait celle-ci : toute civilisation humaine croit (intuitivement?) que si elle se mange elle-même elle finira, fatalement, par ne plus exister : l'être qui se mangerait lui-même deviendrait nonêtre. Une autre question, plus importante à nos yeux, apparaît alors : le cannibalisme serait-il la clef de voûte de la métaphysique ? Une chose est sûre en tout cas : c'est en posant le cannibalisme comme ce qui amènerait fatalement l'extinction, la néantisation de l'être - une sorte de politique de la peur ontologique -, que l'intestin s'est vu rabattu en cerveau et en tube digestif afin, du moins les métaphysiciens le croient-il, de ne pas se manger lui-même - et de disparaître dans le néant. La création de cette organisation complexe en organisme (cerveau qui pense et idéalise, tube digestif qui mange, chie et matérialise) est censé garantir le meilleur rempart contre le risque cannibalique. Voire. 


\section{(V). L'intestin, encore} yeux. Mais, même si nous ne pouvons le percevoir, il n'en est pas moins certain que nous pouvons le sentir. Quand les aborigènes dessinent à même la terre ce qu'ils nomment le chant des pistes (que Bruce Chatwin, dans son roman éponyme, décrit si bien), c'est leurs rêves qui s'exposent sans dualité, leurs rêves/chemins sont tout en même temps l'immatériel des rêves et le matériel de la poussière du désert australien, et expriment une complétude qui semble rejoindre le cri d'Antonin Artaud à la fin de sa pièce Pour en finir avec le jugement de Dieu où il appelle l'homme à enlever tous ses organes, à danser sens dessus dessous, à exposer son corps à l'envers pour découvrir son véritable endroit par delà omote et ura.

lestes pour danser comme Nietzsche ou Artaud, où pouvons-nous le dénicher, cet intestin? Où le trouvera-t-on ce plan de consistance? Justement dans tout ce qui correspond à des intestins qui ne sont pas, pour autant, des tubes digestifs. En effet, tous les intestins ne sont pas en forme de tube. Il y a un intestin dont la forme est plate et ouverte et que l'on connaît tellement bien qu'on a oublié qu'on le connaissait : on a pris l'habitude de l'appeler environnement naturel ou monde (cette fameuse nature dont rêvent les écologistes). Mais là encore, prenons gare aux apparences et, à peine découvert, ne le rabattons pas sitôt : car, en réalité, même si on le voit ouvert et plan, tout ceci n'est qu'une fausse apparence. Cet intestin n'est pas plat, comme n'est pas plate la terre, mais sphérique, comme les scientifiques nous ont appris qu'elle l'était - mais même la sphère est déjà une construction du cerveau trop idéaliste et, pour tenter de le cerner au plus près, nous dirons plutôt que l'intestin est en courbes.

Voilà comment reconnaître un intestin : d'un point de vue topologique, tous les intestins sont des courbes pliées, que nous voyons tantôt repliées en dedans (comme un gant replié sur lui-même : c'est l'intestin animal tel qu'on le connaît), tantôt dépliées en dehors (c'est la sphère, comme la terre). Ces perceptions de l'intestin ne sont donc que des constructions qui nous permettent d'appréhender l'intestin: il serait, par exemple, erroné de dire que la nature environnementale est un intestin sphérique, ouvert qui le différencierait fondamentalement de l'intestin humain, en tube et fermé. En effet, dans tous les cas, et c'est la seconde caractéristique de l'intestin (après sa topologie en courbes), son dehors et son dedans sont toujours liés, il est même la membrane où se lient, se délient et se relient le dedans et le dehors, l'omote et le ura. 
59 L'intestin que nous traquons - ce plan de consistance - est en fait une membrane qui se plie à l'infini, une danse de courbes où intérieur et extérieur dansent aussi, comme dans le «bals musette » du Jugement de Dieu de Artaud, celui où les termes d'endroit et d'envers perdent leur sens. Ainsi se dessine mieux le qualificatif de plan que Guattari-Deleuze utilisent pour nommer cet intestin, en tout cas, si on se méprend pas sur le fait qu'il ne s'agit pas d'un plan au sens d'une feuille (avec un recto et un verso opposés et dualisés, un omote et un ura irréductibles et hétérogènes), mais d'un plan au sens qu'il consiste, et cela malgré son étrange forme.

\section{Un intestin japonais animiste}

60 Jouons une comparaison anthropologique entre le Japon et la France pour le mieux cerner encore, cet intestin. Au Japon, l'intestin est pensé comme un cercle: dans le shintoisme, l'intestin part de la terre pour y retourner. Les kami ou esprits, n'ont jamais « le temps ", après leur venue dans le monde des humains, de devenir autre chose qu'un esprit de la nature - par exemple un Dieu du poly- ou du monothéisme - : un renard, à l'heure du bœuf comme on appelle l'heure des fantômes au pays du soleil levant, se transforme en femme pour séduire un homme mais redevient fatalement, après son forfait, un renard ; la légende d'Ukemochi illustre cela très bien, qui raconte que cet esprit de la nourriture, pour honorer un invité, n'avait qu'à se tourner vers la mer pour que de sa bouche sortent poissons et algues comestibles, vers les rizières pour qu'elle crache du riz bouilli délicieux, vers les forêts pour faire sortir de son tube digestif, en les chiant par l'anus, des animaux près à être consommés.

61 De même Doi Takeo, un psychanalyste japonais, lorsqu'il tente de comprendre la construction subjective de l'individu japonais, construit le concept d'amae pour montrer que du plus jeune âge jusqu'à sa mort, le Japonais se construit en incluant ou en excluant de son cercle subjectif (qui est une sorte de ritournelle existentielle comme le décrit Guattari) tout ce que le cadre social lui permet, ou non, d'y inclure ou d'en exclure - la langue ainsi que les rapports sociaux japonais sont imprégnés de la notion d'uchi-soto qui décrit l'appartenance d'un individu à son propre cercle (uchi signifie dedans mais aussi maison) ou à un cercle extérieur (soto signifie extérieur). Un dernier exemple : le iki, tel que le développe le philosophe Kuki Shûzô, qui correspondrait à ce qu'en français on pourrait appeler le bon goût, et qui, s'il pourrait sembler dans une lecture occidentale superficielle s'opposer au vulgaire, est en fait plus complexe (plus circulaire que cela). Que ce soit les prostitués de l'époque médiévale d'Edo - a priori l'expression la plus basse de la société - qui aient poussé le iki à son paroxysme (ce que les peintres du monde flottant, à travers les estampes de l'Ukiyo-e ont admirablement mis en images, exposant paysages et personnages teintés de ce subtil mélange d'audace raffinée, spontanée, nonchalante et vaguement indifférente, emprunt d'une beauté qui ne cherche par l'être) montre bien que ce qui est pris des bas-fonds, quand bien même il devient iki, ne fait toujours que revenir à autre chose qu'à lui-même, dans un mouvement circulaire infini.

\section{Un intestin français psychanalytique}

62 Ainsi, quand le gouvernement japonais joue la carte de l'isolationnisme (voir la politique raciste de l'État japonais vis-à-vis des Coréens, sa politique d'immigration plus que contrôlée), c'est bien pour pouvoir faire marcher, à lui toute seul, son intestin en cercle. 
Bien différent est l'intestin occidental (Français notamment) qui vise toujours plus haut : sans même parler, évidemment, de l'élévation transcendantale du Dieu des monothéismes, de l'idéal hégélien du Concept devenu l'Être ultime, de la République française qui se glorifie de l'idéal des Droits de l'Homme qu'elle bafoue pourtant régulièrement ( $c f$. la politique d'immigration raciste de l'État français, la création des camps de rétention où sont concentrés les "sans papiers ", les roms et nombre de parias de la société - on voit ici que Japon et France, s'ils se retrouvent avec la même moderne politique migratoire raciste et xénophobe, ne le font pas pour les mêmes raisons, leurs intestins n'ayant pas exactement le même dispositif digestif), le concept d'indifférenciation du bébé d'avec sa mère, portée par une psychanalyse particulièrement tendance en ce tournant de millénaire, aussi simplifiée que simpliste, donne un exemple pénétrant de cette façon dualiste de considérer l'intestin.

Cedévoiement de la psychanalyse a-t-il un autre rêve que d'élever l'homme toujours plus haut, lui qui clame que l'individu est d'abord un intestin indifférencié qui, de bébé identique à sa mère, va s'individualiser en se soumettant à la castration du père tout puissant ? Un bébé qui suce le sein de sa mère serait un bon modèle de cette construction d'un intestin dualiste vertical : il serait tout d'abord, si l'on en croit les discours de la grande majorité des psychanalystes d'enfants, un tube digestif tout juste capable de prolonger le monde qu'est sa mère (en tétant son sein pour en extraire le lait, il ne se différencie pas, dans un premier temps, de ce sein) - stade oral de la première année. C'est dans un second temps qu'il se subjectiverait en individu en reconnaissant que lui et ce sein, ça fait deux : il apprendrait en effet à se différencier du monde résumé jusque là à sa mère pour s'ouvrir (en apprenant à expulser, suivant la dualité de son plaisir/déplaisir, ses selles) à un monde où il se construirait comme différent de l'autre - stade anal de la deuxième et troisième année.

C'est par tout ce branle-bas de combat qu'il impulserait autour de lui (structuration de la famille, droit d'héritage et de succession, rôle du père, réorganisation du travail de la mère, modification, de par sa seule présence, de l'organisation sociale) qu'il modifierait massivement les échanges, les systèmes de production et de distribution de la famille et de la société - complexe d'œdipe de la troisième et quatrième année. Lorsque ce «psychanalisme » - que nous différencions d'une psychanalyse qui peut se dire, comme notre philosophie, de l'intestin - affirme que le bébé, d'un point de vue subjectif, n'est à l'origine pas différencié de sa mère, lorsqu'il soutient qu'il est, avec elle, le monde entier parce qu'il est justement un prolongement du tube digestif de sa mère, et lorsque enfin il affirme qu'il se construit subjectivement (individuellement) en structurant son appareil psychique (qui, même s'il n'est pas, pour cette bouillie pseudo-psychanalytique, superposable au cerveau biologique, correspond assez bien à ce que nous avons appelé jusque là cerveau), lorsque donc le "psychanalisme » invente cette ontogenèse, il ne fait pas autre chose qu'affirmer le double mouvement, vers l'avant et vers l'arrière, que nous avons décrit plus haut: le bébé-maman intestin monde se dualise et construit un monde où, comme sujet individualisé, il apprend à couper le monde en deux, en se structurant comme organisme composé d'un cerveau s'adjoignant un tube digestif qui lui est propre (qui n'est plus celui de sa mère) et qui va lui servir à digérer le reste du monde (qui devient autre) qu'il mange - extension vers l'avant, point différentiel du feu - et dans lequel il rejette ses déchets - extension vers l'arrière, point différentiel de l'eau. Le bébé doit, comme tout individu, faire de ce qu'il mange et de l'endroit où il excrète ses excréments, un autre - interdit du cannibalisme oblige. Autrement dit, cette façon 
particulièrement peu politique de faire de la psychanalyse, ne fait que reproduire le mouvement qui consiste à soumettre la physique à la métaphysique ou plutôt, dans ce cas, la métaphysique à la physique.

\section{Deux premières caractéristiques de l'intestin}

65 À l'image de ce bébé qui devient l'individu occidental, toute l'histoire de l'Occident semble une course sans fin pour atteindre les sommets: comme le roman, cet art architectural et pictural des XI ${ }^{\text {ème }}$ et XII ${ }^{\text {ème }}$ siècles qui fut longtemps, et encore mais de façon progressive plus que progressiste - mais toujours à tort pensons-nous, nous $\mathrm{y}$ reviendrons dans quelques lignes - perçu par la plupart des historiens de l'art que comme le brouillon, le plan d'où émane, sublime nécessairement, le Gothique qui, de flamboyant, explose en baroque où l'intestin, nous dit Deleuze, devient un pli sans fin, se repliant sur lui-même (voir son livre éponyme), comme un bâtiment à deux étages, cerveau au grenier et intestin à la cave. Mais, au-delà des tours en cercle et sans fin ou d'une ascension infinie et verticale, l'intestin, comme l'a bien compris Deleuze, est beaucoup plus simple que cela - et plus complexe à penser par un Japonais ou un Français qui n'est ni aborigène, ni Artaud, ni Nietzsche, ni Bashô.

Nous voyons, par ce petit exercice d'anthropologie comparée auquel nous nous sommes prêtés que, japonais ou français, l'intestin est ici toujours pensé en termes dualistes car, en cercle ou à la verticale, il ne cesse de s'étendre vers l'avant et vers l'arrière. Or il n'y a pas de progression en avant-maintenant-après au sein de l'intestin; et il n'a pas plus de dehors (ce qui est dedans pour un côté de la membrane, est dedans pour l'autre, et vice et versa) qu'il n'a de dedans. L'intestin est-il pour autant un ruban de Möbius (anneau qui ne comporte qu'une seule face, le bord de sa surface compacte étant homéomorphe à un cercle)? Nous ne le croyons pas. Les jeux topologique de Lacan (repris par son élève Anzieu dans son concept de moi-peau (voir son livre éponyme), ce qui permet à ce dernier d'expliquer notamment que le moi, chez le pervers, prend la forme d'un ruban de Möbius, sans dedans ni dehors, ce qui expliquerait sa personnalité suffisamment individualisée pour ne pas pouvoir être qualifiée de psychotique mais pas assez pour pouvoir être qualifiée de névrosé) ne nous aident aucunement à saisir l'intestin que nous cherchons ici.

67 Pour nous, l'intestin ne se laisse pas prendre à une figure mathématique quelle qu'elle soit, et, pour l'instant, nous ne pouvons que l'envisager comme une membrane qui 1) se plie à l'infini, dans l'espace et dans le temps, dans une danse de courbes sans fin et où 2) se lient, se délient et se relient sans fin le dedans et le dehors, l'omote et le ura. Peut-être nous faut-il reprendre, pour l'attraper au vol cet intestin, l'histoire naturelle que nous avions entamée (hydre/thon /homme/chat/bactérie...) et s'intéresser maintenant aux végétaux, espèce où cerveau et tube digestif semblent ne s'être même pas différenciés.

\section{Suite de nos histoires naturelles : l'intestin des végétaux}

C'est en tout cas ce que l'on peut croire si l'on en reste à une analyse superficielle. Car les végétaux aussi ont un intestin - mais peut-on pour autant dire que les végétaux sont euxmêmes un intestin? Regardons. Un arbre par exemple, est composé de racines d'où il absorbe dans la terre les nutriments qui lui sont nécessaires, et rejette, par ses feuilles issues de ses tiges, l'oxygène, déchet de sa digestion, nourriture de cet autre du règne 
végétal qu'est le règne animal. Ainsi, si nous prenons l'analogie du tube digestif (bouche/ tube/anus), on peut dire qu'une plante absorbe ses aliments en prolongeant sa bouche dans la terre et son anus vers le ciel, le stade ultime de cette digestion étant, en hiver, lorsque la digestion s'arrête (que la plante se met au repos), lorsqu'elle laisse tomber ses feuilles mortes à terre, merde qui, comme futur terreau, deviendra aliment pour d'autres plantes. (Une remarque, toute deleuzo-guatttarienne: cet analogie avec le tube digestif ne concerne pas que les arbres mais toutes les plantes en général, y compris celles qui ont vu se transformer leur feuilles en aiguilles, qui ont vu se modifier leurs racines pour puiser les nutriments dans l'air même, ou encore celle qui utilisent le rhizome souterrain ou non - comme organe de réserve mais qui ont néanmoins toujours, ne l'oublions pas, des racines et des tiges). Mais il ne faut pas pousser l'analogie trop loin car, contrairement à l'intestin animal qui ne marche, a priori, que dans un seul sens, cet intestin végétal, lui, marche dans les deux sens : l'anus/tige est aussi une bouche car elle se connecte au soleil (aux photons, excréments de sa complexe et fantastique digestion gazeuse, que cet autre que la terre chie sur elle) pour absorber (manger), par photosynthèse, cette lumière/aliment. Nous voyons donc bien que, dans le règne végétal, s'il existe des tubes digestifs, ceux-ci ont la capacité de se plier pour échanger leurs points d'entrée et de sortie.

\section{Troisième caractéristique de l'intestin}

Mais l'analogie que nous avons proposée entre végétaux et animaux considère encore l'intestin comme un tube et, peut-être, un corps unicellulaire serait l'exemple le plus simple, et le plus évident, de l'aspect possiblement sphérique de l'intestin où anus et bouche sont plus qu'interchangeables, puisqu'ils ne sont pas différenciables (chaque vacuole d'échange qui permet l'entrée ou la sortie des nutriments n'est qu'un prolongement, en dedans ou en dehors, de la membrane cellulaire). Ce que nous voulons montrer, c'est que, sous ce que nous avons pris l'habitude de nommer intestin, il y a en fait une infinité de formes : multiples, en cercles, en tube, avec point d'entrée et de sortie échangeables ou irrémédiablement irréductibles, l'intestin semble prendre toutes les formes possibles pour permettre l'agencement du vivant. Ainsi en tirons-nous la conclusion que cet intestin, s'il se laisser attraper (penser) de ces différentes manières, c'est pour mieux nous laisser deviner quel serait la danse que nous pourrions engager avec lui.

70 Si nous insistions, à propos des végétaux, sur les articulations des aliments et des déchets des plantes avec les animaux (dont l'homme), le soleil, la terre, c'est pour montrer que l'intestin possède, de fait, outre les deux caractéristiques que nous lui avons prêtées (1- il se plie, danse, à l'infini, dans l'espace et dans le temps et 2- il permet les jeux de (de/ re)liaisons de l'endroit/avant (omote) et de l'envers/arrière (ura), une troisième caractéristique: il est plus collectif qu'individuel, en ce sens qu'il est composé de fragment(s), mais de fragments(s) d'un genre particulier - d'où le $s$ entre parenthèse - : les morceaux qui le constituent ne s'agencent pas comme les pièces d'un puzzle qui donnerait, in fine, une image globale de l'unité que serait censé être cet agencement : ils sont plutôt des zones d'intensités qui se forment et se déforment, comme des courants magnétiques qui, en constante variation, orienteraient dans tous les sens possibles un plan de limailles de fer. 
71 Pour l'homme en tant que catégorie biologique, on aura compris que nous considérons son intestin comme en plis, modifiant constamment son dedans et son dehors, et composé par de multiples intestins innombrables et collectif (cette dernière et troisième caractéristique ne tenant pas uniquement, ne nous trompons pas, au fait qu'il contient, qu'il est composé de multiples bactéries sans lesquelles la fonction de digestion du tube digestif serait impossible). De même le globe terrestre peut-il être considéré comme un intestin qualifiable par ces trois caractéristiques (il est un intestin en pli, composé de multiples intestins où dedans et dehors se différencient et s'indifférencient sans cesse). Un modèle mathématique permettrait-il de penser ce que nous venons d'écrire, comme par exemple le modèle des fractales ou encore celui des poupées russes? N'étant pas mathématicien nous ne nous aventurerons pas sur ce terrain et retiendront les leçons philosophiques de Guattari-Deleuze dans Qu'est-ce que la philosophie? lorsqu'ils insistent sur ce qui différencie les productions des scientifiques, des philosophes et des artistes: nous, comme philosophes, nous créons des concepts, et non pas des affects ou des percepts cela nous évitera, de plus, les ires de Sokal ou de Bricmont...

La nature? Elle aussi peut être conceptualisée comme un intestin qui répond aux mêmes critères. Si, malgré tout, prend l'envie au plan de consistance de se laisser prendre au jeu de l'analogie, et qu'on veut penser son aspect collectif comme l'emboitement de poupées russes, ou les strates successives d'un oignon, il faudra alors concevoir des poupées ou des oignons desquels, plus on enlève les peaux ou les poupées les plus superficielles, plus les peaux et les poupées en profondeur ne seront 1) ni forcément plus petites, et 2) ni forcément à l'intérieur : la cellule n'est pas contenu dans l'homme qui serait contenu par la terre elle-même contenue par le cosmos. Si on épluche l'homme, on risque bien de ne pas tomber sur la cellule, mais sur le cosmos: son intérieur ouvre, potentiellement, sur l'extérieur, par des plis complexes, temporels et spatiaux, qui peuvent faire advenir le passé après, le plus grand à l'intérieur du plus petit - même s'il aussi possible, en ouvrant la terre, d'y découvrir, plus petits qu'elle, des êtres humains composés de cellules. Shakespeare ne nous a-t-il pas montré que l'univers tout entier pouvait reposer dans la coquille d'une noix?

\section{(VI). Un intestin par delà...}

73 Ainsi explosent les dualités que nous connaissons et qui répondent à la sacro-sainte règle logique des causes et des effets. Le petit est plus petit que le grand; le dedans est en dedans $\mathrm{du}$ dehors; l'avant est avant l'après; le vivant est plus vivant que le mort, etc. Mais l'intestin ne connait pas de distinction entre la vie et la mort, pas plus qu'il ne se soumet au temps chronologique qui ordonne le temps en passé-présent-futur : fragment(s), il ne fait que danser, re(de)composer. Par-delà vie et mort... Comme nous l'ont enseigné Guattari et Deleuze, l'intestin produit. Que produit-il ? De l'intestin. Même la mort est un produit de l'intestin, car la mort est un changement du processus de la vie de l'intestin. Et, de même, la vie est un produit de l'intestin qui le rend plus mort encore - sans le tuer. Au sein de l'intestin, la mort n'est pas la sortie de la vie et la vie l'état opposé à la mort. Devrions-nous ici utiliser le mot leibnizien de monade pour parler de l'intestin? Non pas, car si l'intestin que nous décrivons est une substance physique qui aspire, mange, évacue, se connecte avec lui-même (c'est-à-dire avec les autres intestins qu'il est aussi), s'il se prolonge indéfiniment, il ne change pas pour autant d'essence. Par-delà essence et substance... 
74 Ainsi, pour nous, l'intestin se veut-il différent de la monade leibnizienne, car la monade a besoin d'un concept métaphysique pour tenir debout (au sens architectural, consistant, du terme) : pour Deleuze une monade a deux étages: le premier est matériel (le corps, physique) et le second étage est métaphysique (Dieu) : les deux sont irréductibles l'un à l'autre et sont les fondations du bâtiment qui est composé par les étages qui le surplombent. Pour Deleuze, le baroque est le plus simple des bâtiments, qui ne comporte que deux étages : le rez-de-chaussée est un étage matériel, avec des fenêtres ; le deuxième étage est immatériel. Le second étage correspondrait, dans notre schéma, au cerveau et le premier étage au matériel du corps (au tube digestif).

Mais notre concept d'intestin, s'il consiste, c'est d'une autre manière que ce que l'architecture baroque propose : l'intestin, lui, quel que soit l'étage d'où on le prenne (et il peut se composer d'une multitude, d'une infinité d'étages), reste de l'intestin et, comme dans le film Cube, où que l'on aille on reste au même endroit en le quittant sans cesse. Le baroque, au contraire, n'est-il pas autre chose que l'envolée, le plus haut possible (plus haut encore que ce que son échelle permettait à Jacob dans son rêve), d'une histoire progressiste (et non une histoire naturelle) qui aurait amené, si on en croit les historiens de ce type d'histoire, la chrétienté occidentale du style roman au style gothique ? Et l'on pense avec dépit aux historiens de l'art recherchant désespérément le chainon manquant, dans une basilique construite entre le $\mathrm{XI}^{\mathrm{e}}$ et le $\mathrm{XII}^{\mathrm{e}}$ siècles, qui montrerait le moment où s'affirme cette dualité, comme d'autres cherchent encore celui qui relie l'animal à l'homme. Et l'on pense avec tristesse à tous les architectes métaphysiciens qui cherchent désespérément comment "tout cela » (la vie, l'être, l'univers...) tient, et qui jettent des anathèmes sur ceux qui ne voient pas, comme eux, la perfection cristalline de leur construction - voir l'obsessionnel Architecte de la trilogie Matrix des frères Washowsky, persuadé, mathématiques à l'appui, du parfait de sa création, et imputant ses dysfonctionnements à l'imperfection humaine, représentée entre autre par une femme noire. L'intestin tel que nous le concevons ? Par-delà gothique et roman.

Nous pensons qu'il ne faut pas nous laisser leurrer et croire aux hiatus que nos sens (raison y compris, n'en déplaise à Kant) nous invite à placer là où il n'y a que continuité soyons plus juste encore : là où il n'y a que l'intestin. L'intestin n'est donc pas l'idéale et raisonnable architecture chère à la métaphysique : l'intestin marche par fragment(s) (3 $3^{\text {ème }}$ caractéristique), danse à l'infini ( $1^{\text {ère }}$ caractéristique) et se plie et se déplie sans cesse (2 $2^{\text {ème }}$ caractéristique). Ces hiatus, tous déclinables en terme de dualités, sont tellement nombreux (ils peuplent en vérité toute notre subjectivité), il y en a tellement que nous pourrions ainsi épingler, que notre livre à venir, et son introduction, risquerait d'être sans fin (ni début) - ne jamais venir. Mais cela ne nous empêchera pas d'en chercher un maximum, au sein de chaque villosité, de chaque bolgia que nous visiterons - dépliant les omote et les ura - afin de les mieux abandonner au fond de notre livre-intestin. Celui-ci sera comme le tapis de Rimbaud (Ma Bohème), ou le taudis (iori) de Bashô, ou la grotte (Zarathoustra avec son aigle et son serpent) de Nietzsche.

77 L'intestin ne se laisse donc pas enfermer dans le bâtiment obscur de la chambre à deux étages, et au confortable et bourgeois sédentarisme auquel cette construction métaphysique invite. Les hommes de l'intestin, profondément nomades, ne connaissent que le voyage car il ne peuvent (et ne veulent) faire autrement que prolonger et étendre l'intestin. Si la monade ferme le monde, l'intestin c'est le on the road de Kerouac. Une route que nous aurions pu prendre, dans ce livre à venir, en jouant, courant et dansant, sur cinq plateaux (termes empruntés à Deleuze et Guattari qui pensent ainsi, dans leur 
livre titré Mille plateaux, dissoudre les chapitres) dont les titres, pensés et titrés comme des dualités à partir de jeux de mots japonais, tenteront de pointer ces dualismes non pour les dépasser (aufhebung), ni pour monter plus haut encore, mais au contraire pour comprendre comment ils constituent le rabattement du plan de consistance que nous nommons intestin et, peut-être, réussir à percevoir la surface sous la profondeur.

$\underline{1}^{\text {er }}$ plateau : homme-intestin ou Surhomme? (Chôjin). En japonais, chôjin peut s'écrire avec les idéogrammes signifiant intestin et signifiant homme, l'ensemble donnant homme-intestin, ou avec les idéogrammes signifiant dépassement et signifiant homme, l'ensemble donnant Surhomme.

$2^{2}{ }^{\text {ème }}$ plateau : intestin en bataille ou Défi ? (chôsen). En japonais, chôsen peut s'écrire avec les idéogrammes signifiant intestin et signifiant bataille, l'ensemble donnant intestin en bataille, ou avec les idéogrammes signifiant rivaliser et signifiant bataille, l'ensemble donnant Défi.

3ème plateau : intestin dépassé ou Transcendance ? (chôetsu). En japonais, chôetsu peut s'écrire avec les idéogrammes signifiant intestin et signifiant aller au delà, l'ensemble donnant intestin dépassé, ou avec les idéogrammes signifiant surpasser et signifiant aller au delà, l'ensemble donnant Transcendance.

4ime plateau : digestion ou Sublimation? (shôka). En japonais, shôka peut s'écrire avec les idéogrammes signifiant éteindre et signifiant transformer, l'ensemble donnant digestion, ou avec les idéogrammes signifiant s'élever et signifiant splendeur, l'ensemble donnant Sublimation.

5 $\underline{\text { ème }}$ plateau : intestin-nature ou Surnature? (chôshizen). En japonais, chôshizen peut s'écrire avec les idéogrammes signifiant intestin et signifiant nature, l'ensemble donnant intestin-nature, ou avec les idéogrammes signifiant surpasser et signifiant nature, l'ensemble donnant Surnature.

\section{(VII). La figure du sacrifié : responsable du rabattement de l'intestin en dualités?}

Une dernière question avant de s'arrêter - provisoirement: si l'intestin s'est dualisé (rabattu avons-nous dit) en cerveau/tube digestif, si le moyen-âge français s'est dualisé en roman/gothique, si l'existence s'est dualisée en mort/vie, si la terre s'est dualisée en nord/sud, est-ce, au bout du compte, la faute de Prométhée qui nous a donné le feu, premier mouvement d'une extension de l'intestin qui a convaincu l'homme que son corps, sa subjectivité, était composée du cerveau en avant et du tube digestif en arrière? Si nous avons fait le lien plus haut entre le feu (foyer) et l'anthropophagie, entre l'eau (toilettes) et la coprophagie (rapport qui montrait que coprophagie et anthropophagie sont des interdits anthropologiques permettant à la civilisation de s'organiser autour du foyer (feu) et des villes (eau)), nous ne pouvons évidemment pas éviter plus longtemps de développer, ne serait-ce qu'un peu, la question complexe du sacrifice - et d'abord celui de Prométhée, attaché à son rocher, expiant son amour des hommes à la face d'un Dieu presque monothéiste (Zeus) n'ayant pour eux que mépris.

\section{Prométhée}

79 Autrement dit, le sacrifice est-il l'axe du rabattement de l'intestin? En donnant le feu aux hommes Prométhée a-t-il, avant sa nièce, ouvert la boite de Pandore et, déjà, a-t-il rabattu l'intestin en cerveau et tube digestif, nous laissant, peut-être à son corps défendant, nous empêtrer dans la toile d'araignée métaphysique où, tapi en son centre, son pire ennemi nous attend pour le Jugement Dernier? Si est bien connu le mythe de 
Prométhée, en l'occurrence la façon dont le titan (auquel pseudo-Apollodore prêtre la création de l'humanité) avait trompé Zeus une première fois en lui laissant choisir, lors du partage des sacrifices que les hommes devaient au roi des dieux, les plus bas morceaux parce qu'il avait eu l'ingénieuse idée de les recouvrir d'un leurre appétissant, et la façon dont il s'était attiré les foudres de Zeus en volant le feu aux dieux et en le donnant aux hommes - geste qui aura une double punition : l'expiation de Prométhée au sommet de sa montagne, son foie, repoussant chaque jour, dévoré par un aigle, et la perte de l'immortalité pour les hommes -, moins connu est le vraie raison du différent entre Zeus et Prométhée.

De la trilogie d'Eschyle ne nous reste que le premier tome, le Prométhée enchainé, mais les deux autres pièces qui lui succédaient (le Prométhée porteur de feu et le Prométhée délivré), et notamment cette dernière, poussaient plus loin le mythe. Prométhée délivré racontait en effet comment Prométhée était délivré par Héraclès, et comment il se réconciliait, in fine, avec Zeus, le sauvant de la chute que le destin lui promettait pourtant. Prométhée, ayant le don de prophétie transmis par sa mère, la titanide Thémis, savait que si Zeus s'unissait avec Thétis dont il était épris, naîtrait de cette union celui qui aurait le pouvoir de renverser le dieu des dieux de son trône. En gage de paix, et pour en finir avec le statut de sacrifié qui était trop lourd à porter, Prométhée aurait ainsi révélé ce fatal savoir à Zeus, lui assurant, au plus haut des cieux, un pouvoir éternel - Prométhée cédait donc. (L'hypothèse d'un Prométhée refusant de livrer le secret à Zeus, et entrainant la chute de ce dernier par les mains du fruit de son union avec Thétis, est d'ailleurs le thème du subversif Prométhée délivré de Percy Bysshe Shelley, qui inverse la conclusion d'Eschyle).

Comme on le voit, Prométhée agit différemment en fonction des légendes, mais une constante reste son rôle dans le rabattement, plus ou moins pérenne, de l'intestin. De cette place médiane entre les dieux (et surtout le Dieu Zeus) et les hommes, Prométhée, le sacrifié par le feu, fait naître les dualités que nous interrogeons: l'homme perd son immortalité et la distinction mort/vie apparaît ; Zeus gagnant ad vitam aeternam le ciel, c'est un nouveau régime du temps qui va apparaître: un temps divin (étage supérieur, idéal) éternel et sans fin, et un temps humain (étage inférieur, matériel), fini et dualisé, rythmé par les travaux et les jours, la vie et la mort. C'est pourquoi nous disons que Prométhée a donné le feu aux hommes pour les aider à prolonger leur intestin - et pourquoi nous émettons l'hypothèse que Prométhée, comme figure du sacrifié, est la condition de ce rabattement humain de l'intestin en un nombre infini de dualités.

\section{Hainuwele}

Une autre légende, polynésienne celle-ci, et abondamment décrite par l'anthropologue allemand Adolf Ellegard Jensen, donne une autre piste de réflexion (parmi de multiples il est vrai) sur les responsables de ces extensions de l'intestin. Un mythe Wemale raconte l'histoire d'Hainuwele (rameau de cocotier), jeune fille qui possédait l'étrange faculté de chier non de la merde mais des trésors, ceci pour la plus grande joie et l'immense enrichissement de son père. Au cours d'une danse cérémonielle, où Hainuwele partagea ces richesses avec les hommes du village, la jalousie les poussa, après avoir profité largement de cette merde/trésor, à la tuer. Son père la découpa alors en une multitude de morceaux et enterra les différentes parties de son corps aux quatre coins de l'île. De chacun naquirent nombres de plantes et notamment des tubercules dont les hommes purent développer la croissance et la culture afin de les manger à leur convenance. En 
offrant l'agriculture à l'homme, Hainuwele a donc déconnecté la bouche et l'anus. En effet, si de son anus naissait une merde ingérable par les hommes (la merde/trésor dont les hommes pouvaient profiter), sa mort et sa transformation en aliments disjoint de la merde (les plantes nées de l'agriculture) marqua une rupture durable - une dualité entre l'aliment d'un côté et la merde de l'autre.

Dans le mythe d'Hainuwele, comme dans celui de Prométhée, le sacrifice a donc une conséquence dans la dualisation: mort/vie; propre/sale; bouche/anus; haut/bas... Hainuwele qui avait nourri les hommes en leur donnant sa merde comme aliment, en étant finalement tuée par les hommes, mutilée en d'innombrables morceaux enfouies dans la terre de l'île, s'est transformée en une multitudes de végétaux où anus et bouche, comme on l'a vu plus haut, même s'ils peuvent échanger leurs rôles, se distinguent fondamentalement. Le sacrifice différencie foncièrement la bouche et l'anus, et cette distinction, comme celle de la vie et de la mort, de l'avant et de l'après que Prométhée a enseigné aux hommes, est devenu leur véritable acte de naissance subjectif, celui de la dualisation de l'intestin.

\section{Fukushima, le rouge-gorge et le Capitalisme}

Aujourd'hui encore cette modalité subjective nous colle véritablement à la peau. Si nous sommes en prise avec le difficile problème de l'énergie nucléaire (feu que l'on dit prométhéen) c'est, nous l'avons vu plus haut, parce que le soleil était également dualisé : source d'énergie salvatrice selon les écologistes (énergie solaire qui pourraient, avec d'autres dites naturelles, remplacer le nucléaire) - et donc source d'aliment : bouche -, il pourrait aussi devenir les toilettes de l'humanité où l'homme ira chier ses déchets nucléaires - anus. Les mythes du feu, au Japon, en France ou ailleurs, sont nombreux mais on y retrouve souvent un oiseau qui va chercher le feu directement dans le soleil pour l'offrir aux hommes, sacrifiant une partie de lui-même en se brûlant - ainsi explique-t-on, en France, la couleur spécifique du rouge gorge. Sacrifice bien coûteux en vérité pour le pauvre oiseau qui enferme l'homme dans des dualités qui l'éloignent de la danse de l'intestin. Est-ce un hasard si, subissant des opérations de la thyroïde, les enfants de Tchernobyl ou ceux, à venir, de Fukushima, se retrouvent, à cause de la cicatrice, avec une gorge rouge ? En se sacrifiant pour permettre à l'homme d'étendre toujours plus son intestin en le dualisant, les Prométhée et les Hainuwele sont comme le sympathique petit oiseau.

On pourrait tenter de tracer, à propos de ces mécanismes d'extension, toute une généalogie (n'est-ce pas la mission que s'est donnée l'anthropologie, et la sociologie à sa suite ?) qui nous aiderait à comprendre la distorsion Fukushima: une généalogie de Prométhée ou du ressentiment de la métaphysique, de la science, de la tragédie, et du capitalisme - une généalogie qui montre comment l'argent peut être dualisé au point qu'une nation (les États-Unis pour ne pas les citer) puisse être, en même temps, la plus endettée du monde et la plus riche du monde. Nous tenterons, à de multiples reprises dans notre livre/intestin, de telles généalogies, mais nous voulons plutôt faire autre chose dans cette introduction. En se rappelant l'histoire de Hainuwele, nous proposons une histoire de la joie, de la physique, du désir, du comique et du non-capitalisme, où l'homme s'amuse, politiquement, à ne plus savoir quelle différence il peut exister entre sa bouche et son anus, ses aliments et sa merde. Où l'homme s'amuse à assumer de vivre 
comme un intestin, ni mort ni vivant (parce que et mort et vivant) - ni homme ni femme, ni animal, ni végétal ni minéral (parce que tous cela à la fois).

Comment nous y prendre? Prenons un dernier exemple: les pays capitalistes ont toujours un problème, qui est un problème d'intestin: cité par Lénine, Rhodes explique que le problème, pour les impérialistes, c'est toujours l'intestin. Pour nous, il entend par là que le Capitalisme, en organisant à l'échelle mondiale d'immenses villes qui consomment de grandes quantités d'aliments et d'énergie qu'elles évacuent sur le globe tout entier, s'est en réalité construit sur la dualité, le rabattement de l'intestin : il s'organise sur la base métaphysique du Cerveau (le Dieu Argent dématérialisé) qui se pense supérieur à l'intestin comme tube digestif (tout ce qui peut exister, des hommes aux plantes, de l'énergie atomique aux nuages, d'un poème à une pomme de terre, et qu'on appelle marchandise) qui lui sert de poubelles, de toilettes. Mais cette logique est profondément erroné, paralogique disent Deleuze-Guattari dans L'Anti-৫Edipe - et ceci même si tout le monde, les métaphysiciens les premiers, sont persuadés de sa logique - car comme nous l'avons écrit, le duo cerveau/tube digestif n'est qu'un rabattement, loin de ce que nous croyons être la vérité de ce qui fait consister fondamentalement l'intestin.

\section{Anti...}

C'est la raison pour laquelle, nous l'avons dit, que nous devrons voyager dans un autre monde, celui de l'instant où Hainuwele et Prométhée sont encore sur le seuil de choisir de créer, ou non, l'humanité - un monde que la psychanalyse de l'intestin appelle, en français inconscient, en allemand, Unbewusste, en anglais, unconscious et en japonais, muishiki. Un monde où la vie et la mort n'existent pas comme dualités, où l'après peut advenir avant l'avant. Si nous n'entamons pas ce voyage au seuil de ce choix, nous ne comprendrons jamais le monde du Capitalisme, cette folie froide du Cerveau/Argent et du tube digestif/marchandises. Nous disons au seuil de leur choix car si l'intestsin s'est vu rabattu, à cause de Prométhée et d'Hainuwele, ces deux-là ont aussi permis à l'intestin d'exister encore dans nos esprits - certains les appellent mythes, d'autres poèmes, d'autres art, et d'autres encore d'autres noms. Encore faut-il accepter de le percevoir : comme les aborigènes nous le rêvent et Artaud nous le poétise. La condition pour cette perception consiste peut-être, simplement, à renverser la réalité et, comme Shelley, comme GuattariDeleuze, d'oser les anti. Anti-Eschyle, anti-đモdipe... anti-ville, anti-réalité... Anti-cerveau, anti-intestin/tube digestif... - Anti-capitalisme.

\section{(...) Une philosophie pragmatique}

Concluons cette introduction: On comprendra que la question, pour nous, en pensant ces deux types de mouvements de prolongation de l'intestin, est de construire une méthode qui ne serait ni de dépassement (le Aufhebung hégélien), ni de recherche de l'origine, mais qui serait, comme le propose Foucault dans son article Marx, Freud, Nietzsche, d'interprétation des interprétations, de trouver une méthode permettant de ne pas concevoir l'intestin ou le cerveau comme transcendant l'un l'autre, mais comme jouant sans cesse à échanger leur rôle (un cerveau qui chie et un tube digestif qui pense). Insistant ainsi, dans cette introduction, sur cette dualité, c'est bien l'intestin, dans sa complétude en fragment(s), que nous traquons. Notre description est très matérielle et pragmatique: il ne s'agit nullement de métaphore. Quand nous disons que l'intestin 
devient un cerveau, ce n'est pas parce qu'il s'idéalise mais simplement parce que il change ses fonctions. Voir une œuvre d'art (Mona Lisa ou le Pavillon d'Or) ou manger un hamburger, lire un tabloïd ou dîner des plus fins mets (shabushabu ou foie gras), tout cela n'est rien de plus que de la digestion. Contre ceux qui, d'ores et déjà, nous taxeraient de nihilisme, nous affirmons que nous ne nions pas l'existence ou l'importance des valeurs, mais qu'elles arrivent, comme nous l'a appris Nietzsche, après.

Certes les intestins humains ne cessent de se prolonger, de façon matérielle (cerveau, dents, villes) ou de façon immatérielle (idées, structures, langages, civilisations). Cependant les intestins immatériels ne sont pas des anges qui travaillent comme les subordonnés d'un Dieu unique d'un quelconque monothéisme. Les intestins matériels sont au contraire les esprits (kami) qui surgissent des pierres, des fantômes issues des arbres et des rivières. Vertical pour les anges, horizontal pour les esprits. C'est la mauvaise connexion de l'intestin immatériel et de l'intestin matériel ou plus précisément la soumission de l'intestin immatériel à l'intestin matériel qui subordonne les productions immatérielles à une fabrication matérielle: les esprits immatériels horizontaux se verticalisent alors pour se soumettre au cerveau matériel. On fait alors rentrer les idées matérielles dans un organe dévolu à cela. Le cerveau se transforme en organe digestif des idées. Ce serait un peu comme regarder son jardin sur une télévision, dans l'écrin de son salon, par vidéosurveillance: les images des fleurs, arbres, ciels et oiseaux ne sont plus des esprits mais deviennent des anges électriques, qui circulent le long des fils ou glissent le long des ondes : la digestion cérébrale, sémiotique devient le niveau final d'un processus de digestion ondulatoire, électronique.

Repensons à nouveau aux deux livres d'Aristote: la métaphysique arrive après la physique : penser (avec son cerveau s'entend) n'est en rien métaphysique : il s'agit d'un processus physique - digestif disons-nous.

Nous répétons que notre concept d'intestin n'est pas une métaphore : il est une phore: il est porteur (phoros en grec) sans méta, comme physique sans méta: le concept d'intestin ne porte pas à travers (méta) quelque chose, il est un porteur lui-même : il porte quelque chose, en dedans et en dehors de lui, ce qu'il porte abolissant la distinction dedans / dehors. Un intestin n'est pas constitué par quelque chose, sauf par lui-même. Même quand il est immatériel, comme une idée, une civilisation, il reste un intestin : mais il n'y a pas de super-intestin, de méta-intestin (qui serait, par exemple, assimilable à un cerveau) mais une multitude d'intestins en connexion les uns avec les autres, chacun prolongeant le précédent et le suivant, dans une danse infini où chacun se différencie, se spécialise tout en restant intestin. L'intestin ne cherche pas la totalisation, sociale par exemple (comme dans les fantasmes d'un contrat social - Rousseau - ou d'un Leviathan - Hobbes). L'intestin ne peut pas céder à la loi naturelle ou au droit naturel : c'est la nature qui se soumet à l'intestin - nous y reviendrons. L'intestin ne cède en rien ses pouvoirs au cerveau : s'il était obligé d'obéir au lois positives, ils tomberait malade ou deviendrait psychotique.

Ce que nous volons accuser, et redresser, c'est cette situation du monde où l'intestin est sommé d'être psychotique par un vol éhonté des idées par le cerveau.

En somme, par-delà les dualismes, nous prônons le pragmatisme. Nous nous revendiquons de la méthode matérialiste quand nous menace l'idéalisme, et nous nous dirons idéalistes devant l'abîme du matérialisme. Nous avons dit, à un moment de cette introduction, une drôle de formule: la surface sous les profondeurs. S'en expliquer ferait 
perdre le suc de ce qui n'est nullement un oxymore ou un paradoxe. Plutôt, nous penserons à Nietzsche qui, à un moment de sa vie, tente de comprendre pourquoi la musique de Wagner, trop idéaliste, trop romantique, trop nihiliste, trop... ne lui convient plus et pourquoi, à ce néant du nord, il préfère la Carmen d'un Bizet du sud. Pris dans d'horribles maux de tête à chercher les raisons d'un tel revirement (Badiou le taxerait sûrement, avec le mépris qui le caractérise, d'« infidèle »), il comprend tout d'un coup, Aurore d'un Gai Savoir, pourquoi Bizet vaut mieux que Wagner : lorsqu'il écoute Wagner, il a mal au ventre. Pas quand il écoute Bizet. Encore une histoire d'intestin. 